\title{
The Inverse Scattering Problem at Fixed Energy for the Three-Dimensional Schrödinger Equation with an Exponentially Decreasing Potential
}

\section{Roman G. Novikov}

CNRS U.R.A. 758, Département de Mathématiques, Université de Nantes, F-44072 Nantes Cedex 03, France, and M.I.T.P.A.N., Warshavskoye sh. 79, kor. 2, 113556, Moscow, Russia

Received 28 April 1993

\begin{abstract}
The relations between the Faddeev functions and the functions of classical scattering theory are found in the complex domain at fixed energy. For the threedimensional case (without assumption of "smallness" of the potential) it is proved that the exponentially decreasing potential is uniquely determined by its scattering amplitude at fixed energy.
\end{abstract}

\section{Introduction}

We consider the three-dimensional Schrödinger equation at fixed positive energy with a real exponentially decreasing potential,

$$
-\Delta \psi+v(x) \psi=E \psi, \quad x \in \mathbb{R}^{3}, \quad E>0 .
$$

The potential $v(x)$ is called exponentially decreasing if

$$
v(x) \in L^{\infty}\left(\mathbb{R}^{3}\right) \text { and } \exists \alpha>0, \exists \beta>0 \text { such that }|v(x)|<\beta e^{-\alpha|x|} .
$$

We consider the Faddeev functions $G(x, k), \psi(x, k), h(k, \ell)$,

$$
\begin{aligned}
& G(x, k)=-\left(\frac{1}{2 \pi}\right)^{3} e^{i k x} \int_{\xi \in \mathbb{R}^{3}} \frac{e^{i \xi x} d \xi}{\xi^{2}+2 k \xi}, \\
& \psi(x, k)=e^{i k x}+\int_{y \in \mathbb{R}^{3}} G(x-y, k) v(y) \psi(y, k) d y, \\
& h(k, \ell)=\left(\frac{1}{2 \pi}\right)^{3} \int_{x \in \mathbb{R}^{3}} e^{-i \ell x} \psi(x, k) v(x) d x,
\end{aligned}
$$

where $k, \ell \in \mathbb{C}^{3}, \ell^{2}=k^{2}=E, \operatorname{Im} \ell=\operatorname{Im} k \neq 0$. 
For the potential $v(x)$ with the property $(0.2)$ we weaken the condition $\operatorname{Im} \ell=$ $\operatorname{Im} k \neq 0$ in the domain of definition of the function $h(k, \ell)$ to $|\operatorname{Im} \ell-\operatorname{Im} k|<\alpha$, $\operatorname{Im} k \neq 0$.

We consider functions $G^{+}(x, \sqrt{E}), \psi^{+}(x, k), f(k, \ell)$ from the classical scattering theory

$$
\begin{aligned}
G^{+}(x, \sqrt{E}) & =-\frac{1}{4 \pi} \frac{e^{i \sqrt{E}|x|}}{|x|}, \quad \sqrt{E}>0 \\
\psi^{+}(x, k) & =e^{i k x}+\int_{y \in \mathbb{R}^{3}} G^{+}(x-y, \sqrt{E}) v(y) \psi^{+}(y, k) d y \\
f(k, \ell) & =\left(\frac{1}{2 \pi}\right)^{3} \int_{x \in \mathbb{R}^{3}} e^{-i \ell x} \psi^{+}(x, k) v(x) d x
\end{aligned}
$$

where $k, \ell \in \mathbb{R}^{3}, \ell^{2}=k^{2}=E$.

Let be $M_{\varrho}=\left\{\left.m\left|m \in \mathbb{C}^{3}, m^{2}=E,\right| \operatorname{Im} m\right|^{2}<\varrho^{2}\right\}, \varrho>0$. For the potential with the property (0.2) we weaken the conditions $k \in \mathbb{R}^{3}, \ell \in \mathbb{R}^{3}, \ell^{2}=k^{2}=E$ in the domains of definition of the functions $\psi^{+}(x, k)$ and $f(k, \ell)$ to $k \in M_{\alpha / 2}, \ell \in M_{\alpha / 2}$. It is well known in this case that $f(k, \ell)$ is a holomorphic function on $M_{\alpha / 2} \times M_{\alpha / 2}$.

In this paper (Proposition 1) we have found the relation between the function $G(x, k)$ and the function $G^{+}(x, \sqrt{E})$ in the form of the concrete realization of the fundamental Euler-Ehrenpreis principle and as a generalization of the Faddeev representation (1.16). (We reproduce in the introduction Propositions 1, 2, 3 and Theorem 1 in the variant B.)

\section{Proposition 1B.}

$$
G(x, k)=-\frac{e^{i \sqrt{E}|x|}}{4 \pi|x|}+\left(\frac{1}{2 \pi}\right)^{3} \int_{m \in R(k)} s(k, m)(|m|)^{-1} e^{i m x} \sigma(d m),
$$

where

$$
\begin{aligned}
& k \in \mathbb{C}^{3}, \quad k^{2}=E>0, \quad \operatorname{Im} k \neq 0 ; \quad R(k)=R_{1}(k) \cup R_{2}(k), \\
& R_{1}(k)=\left\{m \mid m \in \mathbb{C}^{3}, m^{2}=E, \operatorname{Im} m=0, m \operatorname{Im} k \geq 0\right\}, \\
& R_{2}(k)=\left\{m\left|m \in \mathbb{C}^{3}, m^{2}=E, 0<\right| \operatorname{Im} m|\leq| \operatorname{Im} k \mid,\right. \\
& \left.\quad \frac{\operatorname{Im} m}{|\operatorname{Im} m|}=\frac{\operatorname{Im} k}{|\operatorname{Im} k|}\right\} ; \\
& s(k, m)=\pi i \text { for } m \in R_{1}(k), \\
& s(k, m)=\pi \text { for } m \in R_{2}(k) ; \\
& \sigma(d m) \text { is a surface element on } R(k) .
\end{aligned}
$$

Further, using (0.9) we have found the relation between functions $\psi, h$ and $\psi^{+}, f$ as a generalization of the Faddeev equations (1.17), (1.18). 
Proposition 2B. Suppose that the potential $v(x)$ has the property (0.2). Then

$$
\psi(x, k)=\psi^{+}(x, k)+\int_{m \in R(k)} h(k, m) s(k, m)(|m|)^{-1} \psi^{+}(x, m) \sigma(d m),
$$

where $k^{2}=E>0,0<|\operatorname{Im} k|<\alpha / 2$.

Theorem 1B. Suppose that the potential $v(x)$ has the property (0.2). Then the following equation is valid:

$$
h(k, \ell)=f(k, \ell)+\int_{m \in R(k)} h(k, m) s(k, m)(|m|)^{-1} f(m, \ell) \sigma(d m),
$$

where $k^{2}=E>0,0<|\operatorname{Im} k|<\alpha / 2, \ell \in R(k)$.

Further, we have found the relation between the modified Fredholm determinants $\Delta(k), \Delta^{+}(\sqrt{E})$ of Eqs. $(0.4),(0.7)$ and the Fredholm determinant $\tilde{\Delta}(k)$ of Eq. $(0.11)$ as a generalization of the Faddeev equation (1.21).

Proposition 3B. Suppose that the potential $v(x)$ has the property (0.2). Then

$$
\Delta(k)=\Delta^{+}(\sqrt{E}) \tilde{\Delta}(k) \exp \left[\hat{v}(0) \int_{m \in R(k)} s(k, m)(|m|)^{-1} \sigma(d m)\right]
$$

where $k^{2}=E>0,0<|\operatorname{Im} k|<\alpha / 2$,

$$
\hat{v}(p)=\left(\frac{1}{2 \pi}\right)^{3} \int_{x \in \mathbb{R}^{3}} e^{i p x} v(x) d x .
$$

Theorem 1 and Propositions 1-4 are given in Sect. 2.

The results mentioned above were obtained for the purpose of their further application to solving the inverse scattering problem at fixed energy. The statement of this problem consists of the following.

Suppose that the potential $v(x)$ is not known, but we know corresponding to this potential the scattering amplitude $f(k, \ell)$ at fixed energy $E>0$, i.e. under the condition $k, \ell \in \mathbb{R}^{3}, k^{2}=\ell^{2}=E>0$. The determination of the potential $v(x)$ through the function $f(k, \ell)$ at fixed energy is called the inverse scattering problem at fixed energy.

Theorem 2 (announced in [16]). Let a real-valued potential $v(x)$ have the property (0.2). Then the scattering amplitude f for Eq. (0.1) at fixed positive energy E uniquely determines $\hat{v}(p)$ for any $p \in \mathbb{R}^{3}$. (There is no assumption about smallness of norm of the potential $v(x)$ in Theorem 2.)

Remarks. I. It is shown in [14] (Novikov, 1986) that an exponentially decreasing potential for the two-dimensional Schrödinger equation is uniquely reconstructed (under the assumption of smallness of norm of the potential) by scattering amplitude at fixed energy (see, in addition, [17]). A similar result was obtained for the threedimensional case in [15a], (G.M. Henkin and R.G. Novikov, 1986) (see, in addition, [15b]). The corresponding reconstruction procedures from [14, 15, 17] include an 
analytical extension of a real analytical function. (This real analytical function admits certain estimates in the complex domain.)

In [16] for the potential with support in the fixed bounded domain $D \subset \mathbb{R}^{n}$ the reconstruction procedures from [14] and [15] were fundamentally changed. In particular, for the three-dimensional case the uniqueness of the reconstruction of such a potential by the scattering amplitude at fixed energy was proved without assumptions about smallness of norm of the potential. In addition, the reconstruction procedures both for the two-dimensional and for the three-dimensional case are reduced to solving linear integral equations of the different types.

II. The basic results of [16] were presented in the survey [15b] (see in [15b], Sect. 5.2 and Note at the Proofreading) which had appeared in the May-June (1987) number of Uspekhi Mat. Nauk. The basis of our paper [16] (submitted June 10, 1987) is a constructive solution of the problem of the determination of the potential for the multidimensional Schrödinger equation through the Wigner operator (see [7]) at fixed energy. Actually, the same constructive solution to this problem was given later by A.I. Nachmann in [12], (submitted November 2, 1987).

The problem of the determination of the potential for the multidimensional Schrödinger equation by the Wigner operator at fixed energy can be called the Gel'fand problem at fixed energy or the Calderón problem for the Schrödinger equation or an inverse boundary value problem and so on.

In the class of piecewise real analytical functions the uniqueness theorem in such a problem was first given in [22].

In the three-dimensional case in the class of infinitely differentiable functions the global theorem of uniqueness in such a problem was first given in [20].

In the three-dimensional case in the class of bounded measurable functions such a result was given almost simultaneously in [16] and [10].

A suitable historical survey and the survey of results on this problem is given in $[16,21]$.

III. The inverse scattering problem at fixed energy is well investigated for the threedimensional Schrödinger equation with a spherically symmetric potential (see $[2,13]$ ). But we have not discovered, however, the "spherically symmetric" particular case of Theorem 2 in the papers of this series.

Theorem 1, Propositions 3, 4, results of Sect. 3 and results of [15] give, in particular, more constructive proofs of Theorem 2 than the one sketched in [16].

For proving Theorem 2 we use in Sect. 3 the secondary extensions of the scattering data to the complex domain. Earlier the idea of the secondary extension of scattering data to the complex domain was used in [14-17].

The main new ideas of the present paper appeared for the first time in the recent Note of the author [18].

\section{Initial Results}

We consider the three-dimensional Schrödinger equation with an exponentially decreasing potential

$$
-\Delta \psi+v(x) \psi=E \psi, \quad x \in \mathbb{R}^{3} .
$$

The real-valued function $v(x)$ is called exponentially decreasing potential if $v(x) \in$ $L^{\infty}\left(\mathbb{R}^{3}\right)$ and $\exists \alpha>0, \exists \beta>0$ such that

$$
|v(x)|<\beta e^{-\alpha|x|} .
$$


We consider the Faddeev functions $G(x, k), \psi(x, k), h(k, \ell) \Delta(k), G_{\gamma}(x, k)$, $\psi_{\gamma}(x, k), h_{\gamma}(k, \ell), \Delta_{\gamma}(k), \tilde{\Delta}_{\gamma}(k)$. These functions are defined by formulas and Eqs. (1.3)-(1.5), (1.8), (1.9), (1.20)

$$
\begin{aligned}
& G(x, k)=g(x, k) e^{\imath k x}, \quad g(x, k)=-\left(\frac{1}{2 \pi}\right)^{3} \int_{\xi \in \mathbb{R}^{3}} \frac{e^{i \xi x} d \xi}{\xi^{2}+2 k \xi}, \\
& \psi(x, k)=e^{\imath k x}+\int_{y \in \mathbb{R}^{3}} G(x-y, k) v(y) \psi(y, k) d y,
\end{aligned}
$$

where $x \in \mathbb{R}^{3}, k \in \mathbb{C}^{3}, \operatorname{Im} k \neq 0$,

$$
h(k, \ell)=\left(\frac{1}{2 \pi}\right)^{3} \int_{x \in \mathbb{R}^{3}} e^{-i \ell x} \psi(x, k) v(x) d x,
$$

where $k, \ell \in \mathbb{C}^{3}, \operatorname{Im} k=\operatorname{Im} \ell \neq 0, k^{2}=\ell^{2}$. Under the condition (1.2) the restriction $\operatorname{Im} k=\operatorname{Im} \ell \neq 0$ in the domain of the definition of the function $h$ admits weakening to $|\operatorname{Im} k-\operatorname{Im} \ell|<\alpha, \operatorname{Im} k \neq 0$. Here $G(x, k)$ is the Green function of the operator $\Delta+k^{2}, \psi(x, k)$ is a solution of Eq. (1.1) with $E=k^{2}, h(k, \ell)$ is a generalized scattering amplitude.

It is convenient to transform Eq. (1.4) to the form (1.6) and further to the form (1.7),

$$
\mu(x, k)=1+\int_{y \in \mathbb{R}^{3}} g(x-y, k) v(y) \mu(y, k) d y,
$$

where $\psi(x, k)=\mu(x, k) e^{i k x}, \operatorname{Im} k \neq 0$;

$$
(I-B(k)) \psi(x, k)=\sqrt{|v(x)|},
$$

where $I$ is the identity operator, $B(k)$ is an integral operator with the kernel

$$
\begin{aligned}
B(x, y ; k) & =\sqrt{|v(x)|} g(x-y, k) v(y)(|v(y)|)^{-1 / 2}, \\
\varphi(x, k) & =\sqrt{|v(x)|} \mu(x, k), \quad \operatorname{Im} k \neq 0 .
\end{aligned}
$$

Lemma 1 (see $[6,1,15]) .|x| g(x, k)$ is a bounded continuous function on $\left(\mathbb{R}^{3} \backslash 0\right) \times$ $\left(\mathbb{C}^{3} \backslash \mathbb{R}^{3}\right)$.

Under the condition (1.2) due to Lemma 1 the following statement is valid: (1.6) is the linear integral Fredholm equation of the second type with respect to $\mu$ in $L^{\infty}\left(\mathbb{R}^{3}\right)$;

$$
B(x, y ; k) \in L^{2}\left(\mathbb{R}^{3} \times \mathbb{R}^{3}\right), \quad \sqrt{|v(x)|} \in L^{2}\left(\mathbb{R}^{3}\right)
$$

and (1.7) is the linear integral Fredholm equation of the second type with respect to $\varphi$ in $L^{2}\left(\mathbb{R}^{3}\right)$. (It holds under conditions much weaker than (1.2) but we do not want to be distracted from the case of exponentially decreasing potential in this article).

Remark 1. As $|x| \rightarrow \infty$ the functions $G(x, k)$ and $\psi(x, k)$ increase exponentially in the direction forming acute angle with the vector $-\operatorname{Im} k$ (and decrease exponentially in the direction forming acute angle with the vector $\operatorname{Im} k)$. In the paper [4] $\psi(x, k)$ were called the exponentially increasing solutions of the Schrödinger equation. 
The modified Fredholm determinant $\Delta(k)$ of the operator $I-B(k), \operatorname{Im} k \neq 0$ is defined by the formula

$$
\ln \Delta(k)=\operatorname{Tr}(\ln (I-B(k))+B(k))
$$

(see [8] for an informal sense of such a definition).

There exist the following limits:

$$
\begin{aligned}
G_{\gamma}(x, k) & =G(x, k+i 0 \gamma), & \psi_{\gamma}(x, k) & =\psi(x, k+i 0 \gamma), \\
h_{\gamma}(k, \ell) & =h(k+i 0 \gamma, \ell+i 0 \gamma), & \Delta_{\gamma}(k) & =\Delta(k+i 0 \gamma),
\end{aligned}
$$

where $k, \ell, \gamma \in \mathbb{R}^{3}, k^{2}=\ell^{2}, \gamma^{2}=1$.

In addition

$$
\begin{aligned}
& G_{\gamma}(x, k)=\left(\frac{1}{2 \pi}\right)^{3} \int_{\xi \in \mathbb{R}^{3}} \frac{e^{i \xi x} d \xi}{k^{2}-\xi^{2}+i 0(k-\xi) \gamma} \\
& \psi_{\gamma}(x, k)=e^{i k x}+\int_{y \in \mathbb{R}^{3}} G_{\gamma}(x-y, k) v(y) \psi_{\gamma}(y, k) d y \\
& h_{\gamma}(k, \ell)=\left(\frac{1}{2 \pi}\right)^{3} \int_{x \in \mathbb{R}^{3}} e^{-i \ell x} \psi_{\gamma}(x, k) v(x) d x
\end{aligned}
$$

$\Delta_{\gamma}(k)$ is the modified Fredholm determinant of the operator $I-B_{\gamma}(k)$, where $B_{\gamma}(k)$ is an integral operator with the kernel

$$
B_{\gamma}(x, y ; k)=\sqrt{|v(x)|} G_{\gamma}(x-y, k) v(y)(|v(y)|)^{-1 / 2} .
$$

Besides,

$$
\begin{aligned}
G^{+}(x, k) & =G_{k /|k|}(x, k), \quad \psi^{+}(x, k)=\psi_{k /|k|}(x, k), \\
f(k, \ell) & =h_{k /|k|}(k, \ell), \quad \Delta^{+}(k)=\Delta_{k /|k|}(k),
\end{aligned}
$$

where $k, \ell \in \mathbb{R}^{3}$.

Here $f(k, \ell)\left(k^{2}=\ell^{2}=E\right)$ is a scattering amplitude of the Schrödinger equation $(1.1)$

$$
f(k, \ell)=\left(\frac{1}{2 \pi}\right)^{3} \int_{x \in \mathbb{R}^{3}} e^{-i \ell x} \psi^{+}(x, k) v(x) d x
$$

$\psi^{+}(x, k)$ is a solution of the equation

$$
\begin{aligned}
& \psi^{+}(x, k)=e^{i k x}+\int_{y \in \mathbb{R}^{3}} G^{+}(x-y, k) v(y) \psi^{+}(y, k) d y, \\
& G^{+}(x, k)=-\frac{1}{4 \pi} \frac{e^{i \sqrt{E}|x|}}{|x|}, \quad \sqrt{E}=\sqrt{k^{2}}>0
\end{aligned}
$$


$\Delta^{+}(k)$ is the modified Fredholm determinant of Eq. (1.15). The following equations [5] (Faddeev, 1966) are valid:

$$
\begin{aligned}
& G_{\gamma}(x, k)=G^{+}(x, k)+\frac{2 \pi i}{(2 \pi)^{3}} \int_{\xi \in \mathbb{R}^{3}} e^{i \xi x} \theta[(\xi-k) \gamma] \delta\left(\xi^{2}-k^{2}\right) d \xi \\
& \psi_{\gamma}(x, k)=\psi^{+}(x, k)+2 \pi i \int_{\xi \in \mathbb{R}^{3}} h_{\gamma}(k, \xi) \theta[(\xi-k) \gamma] \delta\left(\xi^{2}-k^{2}\right) \psi^{+}(x, \xi) d \xi, \\
& h_{\gamma}(k, \ell)=f(k, \ell)+2 \pi i \int_{\xi \in \mathbb{R}^{3}} h_{\gamma}(k, \xi) \theta[(\xi-k) \gamma] \delta\left(\xi^{2}-k^{2}\right) f(\xi, \ell) d \xi,
\end{aligned}
$$

where

$$
\int_{m \in \mathbb{R}^{3}} \varphi(m) \delta\left(m^{2}-k^{2}\right) d m=\frac{1}{2|k|} \int_{|m|=|k|} \varphi(m) \sigma(d m),
$$

$\theta[t]$ is the Heaviside function.

It is supposed in Eqs. (1.17) and (1.18) that $k$ and $\gamma$ are fixed and $\Delta_{\gamma}(k) \neq 0$.

We consider (1.18) as an equation for definition of the function $h_{\gamma}$ through the function $f$. We can rewrite this equation in the form

$$
\left(I-P_{\gamma}(k)\right) h_{\gamma}(k, \ell)=f(k, \ell)
$$

where $P_{\gamma}(k)$ is a linear integral operator with the kernel

$$
P_{\gamma}(\ell, m ; k)=\frac{\pi i}{|k|} \theta[(m-k) \gamma] f(m, \ell), \quad|m|=|\ell|=|k|,
$$

acting in the space of functions on the sphere of the radius $|k|$. The Fredholm determinant $\tilde{\Delta}_{\gamma}(k)$ of the operator $I-P_{\gamma}(k)$ is defined by the formula

$$
\ln \tilde{\Delta}_{\gamma}(k)=\operatorname{Tr} \ln \left(I-P_{\gamma}(k)\right) \text {. }
$$

The determinants $\Delta_{\gamma}(k)$ and $\tilde{\Delta}_{\gamma}(k)$ are connected by the equation ([6])

$$
\Delta_{\gamma}(k)=\Delta^{+}(k) \tilde{\Delta}_{\gamma}(k) \exp \left[\hat{v}(0) 2 \pi i \int_{m \in \mathbb{R}^{3}} \theta[(m-k) \gamma] \delta\left(k^{2}-m^{2}\right) d m\right]
$$

where

$$
\hat{v}(p)=\left(\frac{1}{2 \pi}\right)^{3} \int_{x \in \mathbb{R}^{3}} e^{i p x} v(x) d x, \quad p \in \mathbb{R}^{3} .
$$

In view of unique solvability of the integral Lippman-Swinger equation for the real-valued potential, $\Delta^{+}(k) \neq 0$, for $k^{2}>0$. Thus [6]

$$
\tilde{\Delta}_{\gamma}(k)=0 \quad \text { iff } \quad \Delta_{\gamma}(k)=0, \quad \text { where }|k|>0 .
$$


In the complex domain the functions $G(x, k), \mu(x, k), h(k, \ell), \Delta(k)$ satisfy $\bar{\partial}$ equations (1.24)-(1.27) and have the properties (1.28)-(1.31) (see [15]).

$$
\begin{aligned}
\frac{\partial}{\partial \bar{k}_{j}} G(x, k) & =-\frac{e^{i k x}}{4 \pi^{2}} \int_{\xi \in \mathbb{R}^{3}} \xi_{j} e^{i \xi x} \delta\left(\xi^{2}+2 k \xi\right) d \xi \\
\frac{\partial}{\partial \bar{k}_{j}} \mu(x, k) & =-2 \pi \int_{\xi \in \mathbb{R}^{3}} \xi_{j} H(k,-\xi) e^{i \xi x} \mu(x, k+\xi) \delta\left(\xi^{2}+2 k \xi\right) d \xi \\
\frac{\partial}{\partial \bar{k}_{j}} H(k, p) & =-2 \pi \int_{\xi \in \mathbb{R}^{3}} \xi_{j} H(k,-\xi) H(k+\xi, p+\xi) \delta\left(\xi^{2}+2 k \xi\right) d \xi, \\
\frac{\partial \Delta(k)}{\partial \bar{k}_{j}} & =2 \pi \Delta(k) \int_{\xi \in \mathbb{R}^{3}} \xi_{j}(H(k, 0)-\hat{v}(0)) \delta\left(\xi^{2}+2 k \xi\right) d \xi
\end{aligned}
$$

where $H(k, p)=h(k, k-p), p \in \mathbb{R}^{3}, p^{2}=2 k p, \operatorname{Im} k \neq 0$,

$$
\begin{aligned}
& \int_{\xi \in \mathbb{R}^{3}} f(\xi) \delta\left(\xi^{2}+2 k \xi\right) d \xi \\
& =\int_{\xi^{2}+2 k \xi=0} f(\xi)\left(4\left|\left(\xi_{1}+\operatorname{Re} k_{1}\right) \operatorname{Im} k_{2}-\left(\xi_{2}+\operatorname{Re} k_{2}\right) \operatorname{Im} k_{1}\right|\right)^{-1}\left|d \xi_{3}\right| .
\end{aligned}
$$

Equations (1.25), (1 26) are considered outside the set of zeros of the determinant $\Delta(k)$,

$$
G(x, k+\xi)=G(x, k), \quad \text { where } \xi \in \mathbb{R}^{3}, \quad \xi^{2}+2 k \xi=0, \quad \operatorname{Im} k \neq 0 .
$$

Let be $V=\left\{k \mid k \in \mathbb{C}^{3} k^{2}=E\right\}$, then for any fixed $x, p \in \mathbb{R}^{3}$ the following formulas are valid:

$$
\begin{array}{lll}
\mu(x, k) \rightarrow 1 & \text { for } \quad k \in V, & |k| \rightarrow \infty ; \\
H(k, p) \rightarrow \hat{v}(p) & \text { for } \quad k \in V_{p}, & |k| \rightarrow \infty,
\end{array}
$$

where $\hat{v}(p)$ is defined by (1.22), $V_{p}=\left\{k \mid k \in V, p^{2}=2 k p\right\}$;

$$
\Delta(k) \rightarrow 1 \text { for } k \in V,|k| \rightarrow \infty .
$$

Historical Remarks. "The part" of $\bar{\partial}$-equations (1.24)-(1.27) (together with boundedness conditions at the infinity) was first discovered by L.D. Faddeev as the following properties of functions $g, \mu, h, \Delta, G_{\gamma}, \psi_{\gamma}, h_{\gamma}, \Delta_{\gamma}$.

I) Let fixed $x, \gamma, k_{\perp}, \ell_{\perp} \in \mathbb{R}^{3}$ be such that $\gamma^{2}=1, \gamma k_{\perp}=\gamma \ell_{\perp}=0, k_{\perp}^{2}=\ell_{\perp}^{2}$. Then with respect to $s$ for $s \in \mathbb{C}, \operatorname{Im} s \geq 0, g\left(x, s \gamma+k_{\perp}\right), \mu\left(x, s \gamma+k_{\perp}\right) \Delta\left(s \gamma+k_{\perp}\right)$, $h\left(s \gamma+k_{\perp}, s \gamma+\ell_{\perp}\right) \Delta\left(s \gamma+k_{\perp}\right)$ are holomorphic functions bounded at infinity; $\Delta\left(s \gamma+k_{\perp}\right)$ is a holomorphic function tending to 1 at infinity.

II) Let be $\gamma \in \mathbb{R}^{3},|\gamma|=1 ; \eta \in \mathbb{R}^{3},|\eta|=1, \eta \gamma=0$;

$$
\left(\eta \nabla_{\gamma}\right)=\sum_{j=1}^{3} \eta_{j}\left(\frac{\partial}{\partial \gamma_{j}}\right) .
$$


Let be $\omega_{\gamma, \eta}(k, \xi)=2 \pi i \eta(\xi-k) \delta\left(\xi^{2}-k^{2}\right) \delta((\xi-k) \gamma)$. Then

$$
\begin{aligned}
\left(\eta \nabla_{\gamma}\right) G_{\gamma}(x, k) & =\frac{1}{(2 \pi)^{3}} \int_{\xi \in \mathbb{R}^{3}} \omega_{\gamma, \eta}(k, \xi) e^{i \xi x} d \xi \\
\left(\eta \nabla_{\gamma}\right) \psi_{\gamma}(x, k) & =\int_{\xi \in \mathbb{R}^{3}} \omega_{\gamma, \eta}(k, \xi) h_{\gamma}(k, \xi) \psi_{\gamma}(x, \xi) d \xi \\
\left(\eta \nabla_{\gamma}\right) h_{\gamma}(k, \ell) & =\int_{\xi \in \mathbb{R}^{3}} \omega_{\gamma, \eta}(k, \xi) h_{\gamma}(k, \xi) h_{\gamma}(\xi, \ell) d \xi \\
\left(\eta \nabla_{\gamma}\right) \Delta_{\gamma}(k) & =-\Delta_{\gamma}(k) \int_{\xi \in \mathbb{R}^{3}}\left(h_{\gamma}(\xi, \xi)-\hat{v}(0)\right) \omega_{\gamma, \eta}(k, \xi) d \xi
\end{aligned}
$$

Equations (1.33), (1.34) are considered outside the set of zeros of the determinant $\Delta_{\gamma}(k)$.

$\bar{\partial}$-equations $(1.24),(1.25),(1.26)$ were first obtained in [1]. The multidimensional $\bar{\partial}$-equations of the type (1.24)-(1.26) were first discovered in [11] and [1]. (In [15a] $\bar{\partial}$-equations (1.24)-(1.26) were obtained under influence of [11] but independently of [1]).

$\bar{\partial}$-equation (1.27) was first obtained in [15] (and no $\bar{\partial}$-equation of that type was known before).

The formula (1.29) (for $E=0$ ) was first noted and used in [1].

The formula (1.30) was first found in [15a]. It is important in particular, in this formula that $\forall p \in \mathbb{R}^{3} \quad V_{p}$ is not empty and is unbounded. (For the two-dimensional case only $V_{0}$ has such a property.) First, the formula of the type (1.30) was given (formally) in [11] for the equation

$$
\left(\sigma \frac{\partial}{\partial t}+\Delta-v(t, x)\right) \psi=0 .
$$

The proof of the formulas (1.29), (1.30) and of the formula of the type (1.31) for $v(x) \in C_{4}^{(4)}\left(\mathbb{R}^{3}\right)$ is given in [15].

The formulas (1.29)-(1.31) are also valid for the case $v(x)(1+|x|)^{3+\varepsilon} \in L^{\infty}\left(\mathbb{R}^{3}\right)$, where $\varepsilon>0$ is fixed. It had been already paid attention to this fact (see [16]). These formulas are the consequences of the uniform estimates for the Green function $g(x, k)$ (see Sect. 8).

\section{The Extension of the Faddeev Equations (1.16), (1.17), (1.18), (1.21) to the Complex Domain A}

Consider the two-dimensional complex manifold

$$
M_{\varrho}=\left\{\left.m\left|m \in \mathbb{C}^{3}, m^{2}=E,\right| \operatorname{Im} m\right|^{2}<\varrho^{2}\right\}, \quad E>0, \varrho>0 .
$$

Lemma 2. $\partial M_{\varrho} \approx S O(3)$ and diffeomorphism is given by the formula

$$
\left[\left(E+\varrho^{2}\right)^{-1 / 2} \operatorname{Re} m, \varrho^{-1} \operatorname{Im} m, \varrho^{-1}\left(E+\varrho^{2}\right)^{-1 / 2} \operatorname{Re} m \times \operatorname{Im} m\right]=g,
$$

where $m \in \partial M_{\varrho}, g \in S O(3), \operatorname{Re} m \times \operatorname{Im} m$ is a vector product. 


\section{Proposition 1A.}

$$
\underset{k \in \partial M_{\varrho}}{G(x, k)}=-\frac{e^{i \sqrt{E}|x|}}{4 \pi|x|}+\frac{1}{(2 \pi)^{3}} \int_{m \in \partial M_{r}} \chi(k, m) e^{i m x} \sigma(d m)
$$

(here $0<\varrho, 0<r, G(x, k)$ is defined by (1.3), $\sigma(d m)$ is an element of volume on $\left.\partial M_{r}\right)$, where

a) if $\varrho<r$ then $\chi(k, m)$ is a continuously differentiable function on (the direct product) $\partial M_{\varrho} \times \partial M_{r}$.

b) if $\varrho=r$ then $\chi(k, m)$ is a continuously differentiable function on $\left(\partial M_{\varrho} \times \partial M_{\varrho}\right) \backslash S_{\varrho}$, where

$$
S_{\varrho}=\left\{(k, m) \mid(k, m) \in \partial M_{\varrho} \times \partial M_{\varrho}, \operatorname{Im} k=\operatorname{Im} m\right\}
$$

and at fixed $k \quad \chi(k, m) \in L^{1}\left(\partial M_{\varrho}\right)$.

c) if $\varrho>r$ then for fixed $k$ the second term in the right-hand side of (2.2) has the sense of the expression $(2 \pi)^{-3} \chi_{k}\left(e^{i m x}\right)$, where $e^{i m x}$ is a function on $\partial M_{r} \cdot \chi_{k}$ is a linear bounded functional on the space $\left.\mathcal{O}\left(\bar{M}_{\varrho}\right)\right|_{\partial M_{r}}$ (holomorphic functions in $\bar{M}_{\varrho}$ restricted to $\partial M_{r}$ ) such that

$$
\chi_{k}(f)=\int_{m \in \partial M_{\varrho}} \chi(k, m) \varphi_{m}(f) \sigma(d m), \text { where } \varphi_{m}(f)=f(m) .
$$

For $\varrho \leq r$ the function $\chi(k, m)$ is defined up to a function $\chi^{\prime}(k, m)$ (with the properties $\mathrm{a}, \mathrm{b}$ ) such that

$$
\int_{m \in \partial M_{r}} \chi^{\prime}(k, m) f(m) \sigma(d m)=0, \quad k \in \partial M_{\varrho}
$$

for any function $f(m)$ admitting an extension to the holomorphic function in $\bar{M}_{r}$. $\chi \sim \chi+\chi^{\prime}$.

The method of finding a concrete function $\chi$ is given in Sect. 4 .

It is possible to consider this method and Proposition 1 as a constructive version of the Ehrenpreis fundamental principle [3] in the concrete situation.

Proposition 2A. Suppose, that the potential $v(x)$ has the property (1.2) then

$$
\underset{\substack{\psi \\ k \in \partial M_{\varrho}}}{(x, k)}=\psi^{+}(x, k)+\int_{m \in \partial M_{r}} h(k, m) \chi(k, m) \psi^{+}(x, m) \sigma(d m),
$$

where $0<\varrho<\alpha / 2,0<r<\alpha / 2$.

Theorem 1A. Suppose, that the potential $v(x)$ has the property (1.2). Then the following equation is valid:

$$
\underset{\substack{\ell \in \partial M_{r} \\ h(k, \ell)}}{h \in f(k, \ell)+} \int_{m \in \partial M_{r}} h(k, m) \chi(k, m) f(m, \ell) \sigma(d m),
$$

where $0<|\operatorname{Im} k|<\alpha / 2,0<r<\alpha / 2$.

In (2.3) and (2.4) the function $\psi(x, k)$ is the exponentially increasing Faddeev solution of $(1.1)$; the function $\psi^{+}(x, k)$ is the solution of (1.15) such that 
$\sqrt{|v(x)|} \psi^{+}(x, k) \in L^{2}\left(\mathbb{R}^{3}\right) ; h(k, \ell)$ is defined on $M_{\alpha / 2} \times M_{\alpha / 2}$ by (1.5); $\chi$ is a function from Proposition 1A; $f(k, \ell)$ is defined on $M_{\alpha / 2} \times M_{\alpha / 2}$ as the holomorphic extension from $S^{2} \times S^{2}$ of the scattering amplitude (here $S^{2}=\{m \mid m \in$ $\left.\left.\mathbb{C}^{3}, m^{2}=E, \operatorname{Im} m=0\right\}\right)$.

It is supposed in Eqs. (2.3) and (2.4) that $k$ is fixed and $\Delta(k) \neq 0$ [where $\Delta(k)$ is defined by (1.8)].

We shall consider (2.4) as an equation for determining the function $h$ through the function $f$. This equation can be rewritten in the form

$$
(I-P(k, r)) h(k, \ell)=f(k, \ell), \quad \ell \in \partial M_{r},
$$

where $0<|\operatorname{Im} k|<\alpha / 2,0<r<\alpha / 2 . P(k, r)$ is a linear integral operator with the kernel

$$
P(\ell, m ; k, r)=\chi(k, m) f(m, \ell), \quad \ell, m \in \partial M_{r},
$$

acting in the space of functions on $\partial M_{r}$.

For $|\operatorname{Im} k|<r, P(\ell, m ; k, r)$ is a continuously differentiable function on $\partial M_{r} \times \partial M_{r}$, for $|\operatorname{Im} k|=r P(k, r)$ is a linear compact operator in $C\left(\partial M_{r}\right)$.

The Fredholm determinant $\tilde{\Delta}(k)$ of the operator $I-P(k, r)$ is defined by the formula

$$
\ln \tilde{\Delta}(k)=\operatorname{Tr} \ln (I-P(k, r)), \quad \text { where } \quad 0<|\operatorname{Im} k|<r .
$$

Lemma 3. $\tilde{\Delta}(k)$ defined by (2.6) does not depend on $r$.

Proposition 3A. Suppose, that the potential $v(x)$ has the property (1.2) then

$$
\Delta(k)=\Delta^{+}(k) \tilde{\Delta}(k) \exp \left[\hat{v}(0) \int_{m \in \partial M_{r}} \chi(k, m) \sigma(d m)\right],
$$

where $0<|\operatorname{Im} k|<r<\alpha / 2, \Delta^{+}(k)$ depends only on $E=k^{2}$ (and it is the same as in (1.21).

Proposition 4A. Let $f$ be a scattering amplitude of the potential $v(x)$ with the property (1.2); $0<|\operatorname{Im} k| \leq r ; k$ is fixed; $\Delta(k)$ is defined by the formula (1.8). Then Eq. (2.4) is uniquely solvable with respect to $h$ in $C\left(\partial M_{r}\right)$ iff $\Delta(k) \neq 0$. (For $0<r<\varrho=|\operatorname{Im} k|$ (2.4) is uniquely solvable in the space $\left.\mathcal{O}\left(\bar{M}_{\varrho}\right)\right|_{\partial M_{r}}$ defined in Proposition 1A iff $\Delta(k) \neq 0)$.

The Extension of the Faddeev Equations (1.16), (1 17), (1.18), (1.21) to the Complex Domain B

Let $k \in \mathbb{C}^{3}, k^{2}=E>0, \operatorname{Im} k \neq 0$. Consider in $\mathbb{C}^{3}$ the two-dimensional real manifold $R(k)$ depending on $k$ :

$$
R(k)=R_{1}(k) \cup R_{2}(k)
$$

where

$$
\begin{gathered}
R_{1}(k)=\left\{m \mid m \in \mathbb{C}^{3}, m^{2}=E, \operatorname{Im} m=0, m \operatorname{Im} k \geq 0\right\}, \\
R_{2}(k)=\left\{m\left|m \in \mathbb{C}^{3}, m^{2}=E, 0<\right| \operatorname{Im} m|\leq| \operatorname{Im} k \mid\right. \\
\left.\frac{\operatorname{Im} m}{|\operatorname{Im} m|}=\frac{\operatorname{Im} k}{|\operatorname{Im} k|}\right\} .
\end{gathered}
$$


Let $e_{1}=\operatorname{Re} k /|\operatorname{Re} k|, e_{2}=\operatorname{Im} k /|\operatorname{Im} k|, e_{3}=\operatorname{Re} k \times \operatorname{Im} k /(|\operatorname{Re} k||\operatorname{Im} k|)$. Let $D$ be a disk of the radius $\sqrt{E}+|\operatorname{Im} k|$ with the polar coordinates $r$ and $\varphi$.

Lemma 4. $R(k) \approx D$, and the homeomorphism is given by the formulas (2.11), (2.12)

$$
\begin{aligned}
& \operatorname{Re} m=\sqrt{E-(\sqrt{E}-r)^{2}}\left(\cos \varphi e_{1}+\sin \varphi e_{3}\right)+(\sqrt{E}-r) e_{2}, \\
& \operatorname{Im} m=0
\end{aligned}
$$

for $0 \leq r \leq \sqrt{E}$,

$$
\begin{aligned}
& \operatorname{Re} m=\sqrt{E+(r-\sqrt{E})^{2}}\left(\cos \varphi e_{1}+\sin \varphi e_{3}\right), \\
& \operatorname{Im} m=(r-\sqrt{E}) e_{2}
\end{aligned}
$$

for $\sqrt{E} \leq r \leq \sqrt{E}+|\operatorname{Im} k|$.

Let $\sigma(d m)$ be a surface element on $R(k)$. Then

$$
\begin{aligned}
& \sigma(d m)=\sqrt{E} d r d \varphi \text { for } m \in R_{1}(k), \\
& \sigma(d m)=\sqrt{E+2(r-\sqrt{E})^{2}} d r d \varphi \text { for } m \in R_{2}(k) .
\end{aligned}
$$

In addition, $|m|=\sqrt{E}$ for $m \in R_{1}(k),|m|=\sqrt{E+2(r-\sqrt{E})^{2}}$ for $m \in R_{2}(k)$. Let $s(k, m)=\pi i$ for $m \in R_{1}(k), s(k, m)=\pi$ for $m \in R_{2}(k)$.

\section{Proposition $1 B$.}

$$
G(x, k)=\frac{-e^{i \sqrt{E}|x|}}{4 \pi|x|}+\frac{1}{(2 \pi)^{3}} \int_{m \in R(k)} s(k, m)(|m|)^{-1} e^{i m x} \sigma(d m),
$$

where $\operatorname{Im} k \neq 0$.

Proposition 2B. Suppose, that the potential $v(x)$ has the property (1.2), then

$$
\psi(x, k)=\psi^{+}(x, k)+\int_{m \in R(k)} h(k, m) s(k, m)(|m|)^{-1} \psi^{+}(x, m) \sigma(d m),
$$

where $0<|\operatorname{Im} k|<\alpha / 2$.

Theorem 1B. Suppose, that the potential $v(x)$ has the property (1.2). Then the following equation is valid

$$
h(k, \ell)=f(k, \ell)+\int_{m \in R(k)} h(k, m) s(k, m)(|m|)^{-1} f(m, \ell) \sigma(d m),
$$

where $0<|\operatorname{Im} k|<\alpha / 2, \ell \in R(k)$.

The functions $G, \psi, \psi^{+}, h, f$ in (2.13), (2.14), (2.15) are the same as in (2.2), (2.3), (2.4). It is supposed in Eqs. (2.14) and (2.15) that $k$ is fixed and $\Delta(k) \neq 0$ (where $\Delta(k)$ is defined by (1.8)). We shall consider (2.15) as the equation for the determination of the function $h$ through the function $f$. This equation can be rewritten in the following form:

$$
(I-P(k)) h(k, \ell)=f(k, \ell), \quad \ell \in R(k),
$$


where $P(k)$ is a linear integral operator with the kernel

$$
P(\ell, m ; k)=s(k, m)(|m|)^{-1} f(m, \ell), \quad \ell, m \in R(k),
$$

acting in the space of functions on $R(k)$.

The Fredholm determinant $\tilde{\Delta}(k)$ of the operator $I-P(k)$ is defined by the formula

$$
\ln \tilde{\Delta}(k)=\operatorname{Tr} \ln (I-P(k)),
$$

where $\operatorname{Im} k \neq 0$.

Lemma 5. $\tilde{\Delta}(k)$ defined by (2.16) coincides with $\tilde{\Delta}(k)$ defined by (2.6).

Proposition 3B. Suppose, that the potential $v(x)$ has the property (1.2), then

$$
\Delta(k)=\Delta^{+}(k) \tilde{\Delta}(k) \exp \left[\hat{v}(0) \int_{m \in R(k)} s(k, m)(|m|)^{-1} \sigma(d m)\right],
$$

where $0<|\operatorname{Im} k|<\alpha / 2 ; \Delta^{+}(k)$ depends only on $E=k^{2}$ and it is the same as in (1.21).

Proposition 4B. Let $f$ be a scattering amplitude of the real-valued potential $v(x)$ with the property (1.2); $0<|\operatorname{Im} k|<\alpha / 2$; let $\Delta(k)$ be defined by the formula (1.8). Then Eq. (2.15) is uniquely solvable with respect to $h$ in $C(R(k))$ iff $\Delta(k) \neq 0$.

Theorem 1 and Propositions 3, 4 bring to the following scheme of the reconstruction of the (three-dimensional) potential decreasing more rapidly than any exponent through its scattering amplitude at fixed energy.

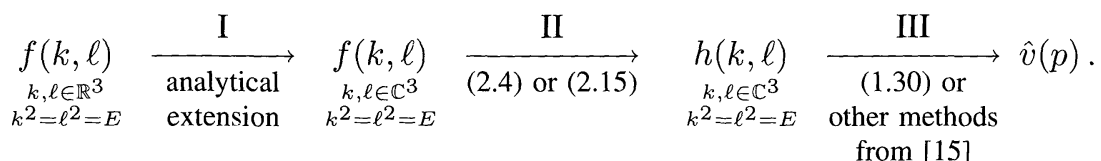

\section{The Double Extension to the Complex Domain and the Global Uniqueness Theorem}

Theorem 2. Let the real-valued potential $v(x)$ have the property (1.2). Then the scattering amplitude $f$ for Eq. (1.1) at fixed positive energy $E$ uniquely determines $\hat{v}(p)$ for any $p \in \mathbb{R}^{3}$.

(There is no assumption about smallness of norm of the potential $v(x)$ in Theorem 2.)

The result of Theorem 2 remains valid if we replace the condition for the potential to be real-valued by the condition $\Delta^{+} \neq 0$ at fixed $E$. Here $\Delta^{+}$is the Fredholm modified determinant of Eq. (1.15).

It is sufficient to prove the result of Theorem 2 for all $p$ such that $p^{2}<4 E$. Besides, it is sufficient to carry out the proof for the case $p=(0,0, \tau)$, where $0<\tau<2 \sqrt{E}$.

Consider a complex curve in $\mathbb{C}^{3}, k^{2}=E, p^{2}=2 k p, k \in \mathbb{C}^{3}$. Introduce on this curve coordinate $\lambda$,

$$
k_{1}=\left(\lambda+\frac{1}{\lambda}\right) \frac{\sqrt{E \tau}}{2}, \quad k_{2}=\left(\frac{1}{\lambda}-\lambda\right) \frac{i \sqrt{E \tau}}{2}, \quad k_{3}=\frac{\tau}{2},
$$

where $E_{\tau}=E-\frac{\tau^{2}}{4}$. 
Let, further, $|\lambda| \geq 1$. Consider the functions $g(x, \lambda, \bar{\lambda})=g(x, k(\lambda)), \Delta(\lambda, \bar{\lambda})=$ $\Delta(k,(\lambda)), h(\lambda, \bar{\lambda})=h(k(\lambda), \ell(\lambda))$, where $\ell(\lambda)=k(\lambda)-p$ and $h(\lambda, \bar{\lambda})=$ $\Delta(\lambda, \bar{\lambda}) \times h(\lambda, \bar{\lambda})$. At fixed $x$ the function $g(x, \lambda, \zeta)$ is a single-valued holomorphic function in the domain

$$
|\lambda| \geq 1, \quad|\zeta| \geq 1, \quad \operatorname{Re}(\lambda \zeta) \geq 0
$$

Besides,

$$
|g(x, \lambda, \zeta)-g(x, \lambda, \bar{\lambda})|<C|\zeta-\bar{\lambda}| \exp (D|\zeta-\bar{\lambda}||x|)
$$

where $C$ and $D$ are constants depending on $E$. As a consequence, for the potential with the property (1.2), $\Delta(\lambda, \zeta)$ and $h(\lambda, \zeta)$ are holomorphic functions in the domain

$$
|\lambda| \geq 1, \quad|\zeta| \geq 1, \quad \operatorname{Re}(\lambda \zeta) \geq 0, \quad|\xi-\bar{\lambda}|<\frac{\alpha}{2 D}
$$

On the other hand, $\Delta(\lambda, \bar{\lambda}) \rightarrow 1$ for $|\lambda| \rightarrow \infty$,

$$
h(\lambda, \bar{\lambda}) \rightarrow \hat{v}(p) \text { for } \quad|\lambda| \rightarrow \infty .
$$

If

$$
0 \leq|\lambda|-1<\frac{\alpha}{2 \sqrt{2 E_{\tau}}}
$$

then $|\operatorname{Im} k(\lambda)|<\alpha / 2$. Thus, under the condition (3.6) the equation (2.4) for $k=k(\lambda)$ holds. If, in addition, $\Delta(\lambda, \bar{\lambda}) \neq 0$ then this equation is uniquely solvable and the function $h(k(\lambda), \ell),|\operatorname{Im} \ell|=|\operatorname{Im} k(\lambda)|$ is uniquely defined by the scattering amplitude at fixed energy.

Thus, the scattering amplitude at fixed energy determines uniquely the function $h(\lambda, \bar{\lambda})$ for all $\lambda$ in the ring (3.6), where $\Delta(\lambda, \bar{\lambda}) \neq 0$.

Note, that a set of such $\lambda$ is open and everywhere dense in this ring.

In order to complete the proof of Theorem 2 it remains to note that the meromorphic function $h(\lambda, \bar{\lambda})$ is uniquely extended from such a set (and, in general, from the neighborhood of any point $\lambda_{0}$ (where $\Delta\left(\lambda_{0}, \bar{\lambda}_{0}\right) \neq 0$ ) in this ring) to all domain 3.4. Taking into account (3.5) we obtain that the scattering amplitude at fixed energy uniquely determines $\hat{v}(p)$.

\section{The Proof of Proposition 1}

We shall use the following results:

1) The initial formula (1.16) will be necessary.

2) Let $E$ be a fixed real number, then (see [15])

$$
\begin{aligned}
G(x, k) & =\overline{G(x, k)}, \quad k^{2}=E, \operatorname{Im} k \neq 0 \\
\left.\bar{\partial} G\right|_{k^{2}=E} & =\left.\frac{-1}{16 \pi^{2}|\operatorname{Im} k|} \sum_{\jmath=1}^{3} c_{j}(x, k) d \bar{k}_{\jmath}\right|_{k^{2}=E}
\end{aligned}
$$


where

$$
\begin{aligned}
& c(x, k)=I_{1}(x, k) \operatorname{Re} k+I_{2}(x, k) \frac{\operatorname{Re} k \times \operatorname{Im} k}{|\operatorname{Im} k|}, \\
& I_{1}(x, k)=\int_{-\pi}^{\pi}(\cos \varphi-1) \exp (i \eta(k, \varphi) x) d \varphi \\
& I_{2}(x, k)=\int_{-\pi}^{\pi}(\sin \varphi) \exp (i \eta(k, \varphi) x) d \varphi \\
& \eta(k, \varphi)=\cos \varphi \operatorname{Re} k+\sin \varphi \frac{\operatorname{Re} k \times \operatorname{Im} k}{|\operatorname{Im} k|}+i \operatorname{Im} k ; \\
&\left.d_{k} G\right|_{k^{2}=E}=\left.\left(\bar{\partial}_{k} G+\partial_{k} G\right)\right|_{k^{2}=E}=\left.\frac{c(x, k) d \bar{k}+\overline{c(x, k)} d k}{-16 \pi^{2}|\operatorname{Im} k|}\right|_{k^{2}=E}, \\
& \operatorname{Im} k \neq 0 .
\end{aligned}
$$

3) Let $R(\zeta)$ be a real smooth function on $\mathbb{C}^{n} ; D=\left\{\zeta \mid \zeta \in \mathbb{C}^{n}, R(\zeta)<0\right\}$ be a convex domain; $s(\zeta)$ be a holomorphic function on $\mathbb{C}^{n} ; V=\left\{\zeta \mid \zeta \in \mathbb{C}^{n}, s(\zeta)=\right.$ $0, R(\zeta)<0\}$ be an intersection of the convex domain with the hypersurface; $F(\zeta)$ be a holomorphic function on $\bar{V}$. Then the following formula of the Cauchy-FantappieLeray type is valid (see $[9,19])$ :

$$
\left.\underset{\eta \in V}{F(\eta)}=\frac{(n-1) !}{(2 \pi i)^{n-1}} \int_{\partial V} F(\zeta) \frac{\operatorname{det}[P(\zeta, \eta), Q(\zeta) \overbrace{\bar{\partial}_{\zeta} Q(\zeta), \ldots, \bar{\partial}_{\zeta} Q(\zeta)}^{n-2}}{(Q(\zeta)(\zeta-\eta))^{n-1}} \wedge(d s(\zeta)\rfloor d \zeta\right),
$$

where

$$
s(\eta)-s(\zeta)=P(\zeta, \eta)(\zeta-\eta), \quad Q(\zeta)=\partial R(\zeta) / \partial \zeta
$$

We shall use formula (4.4) for the case $V=M_{\varrho}$. If $M_{\varrho}$ is defined by (2.1) then (4.4) takes the form

$$
\left.\underset{\eta \in M_{\varrho}}{F(\eta)}=-\frac{1}{2 \pi^{2}} \int_{\zeta \in \partial M_{\varrho}} F(\zeta) \frac{\operatorname{det}[\eta+\zeta, \bar{\zeta}-\zeta, d \bar{\zeta}]}{((\bar{\zeta}-\zeta)(\zeta-\eta))^{2}}(2 \zeta d \zeta\lrcorner d \zeta_{1} \wedge d \zeta_{2} \wedge d \zeta_{3}\right) .
$$

The proof of Proposition 1A is based on the following scheme of calculation of the function $\chi\left(k^{\prime}, \zeta\right)$ : Let $k^{\prime}$ be a fixed point in $\partial M_{\varrho}$. Then

$$
G\left(x, k^{\prime}\right)=G(x, k(+0))+\int_{+0}^{\varrho} d G(x, k(\tau))
$$

where

$$
k(\tau)=\sqrt{E+\tau^{2}} \frac{\operatorname{Re} k^{\prime}}{\left|\operatorname{Re} k^{\prime}\right|}+i \tau \frac{\operatorname{Im} k^{\prime}}{\left|\operatorname{Im} k^{\prime}\right|}, \quad 0 \leq \tau \leq \varrho .
$$


The following formulas are valid:

$$
\begin{aligned}
G(x, k(+0)) & =G_{\gamma^{\prime}}(x, k(0)) \\
& =-\frac{e^{i \sqrt{E}|x|}}{4 \pi|x|}+\frac{i}{(2 \pi)^{2}} \int_{\eta \in \mathbb{R}^{3}} e^{i \eta x} \theta\left(\eta \gamma^{\prime}\right) \delta\left(\eta^{2}-E\right) d \eta
\end{aligned}
$$

where $\gamma^{\prime}=\operatorname{Im} k^{\prime} /\left|\operatorname{Im} k^{\prime}\right|$

$$
\begin{aligned}
\int_{+0}^{\varrho} d G(x, k(\tau)) & =\operatorname{Re} \int_{+0}^{\varrho} \frac{c(x, k(\tau)) d \bar{k}(\tau)}{-8 \pi^{2}|\operatorname{Im} k(\tau)|}=\operatorname{Re} \int_{0}^{\varrho} \frac{I_{1}(x, k(\tau)) d \tau}{-8 \pi^{2}} \\
& =\frac{1}{8 \pi^{2}} \operatorname{Re} \int_{-\pi}^{\pi} \int_{0}^{\varrho} \exp (i \eta(k(\tau), \varphi) x) d \tau d \varphi \\
& =\frac{1}{8 \pi^{2}} \int_{-\pi}^{\pi} \int_{0}^{\varrho} \exp (i \eta(k(\tau), \varphi) x) d \tau d \varphi
\end{aligned}
$$

where $e^{i \eta x}$ in (4.8), (4.9) admits such a representation

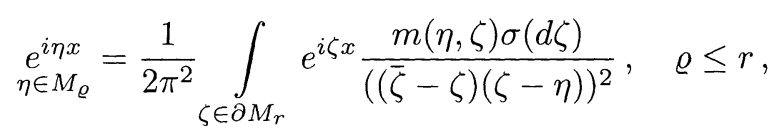

$\sigma(d \zeta)$ is an element of the volume on $\partial M_{r}, m(\eta, \zeta)$ is a linear function of $\eta$ such that

$$
\begin{aligned}
& \left.i \operatorname{det}[\eta+\operatorname{Re} \zeta, \operatorname{Im} \zeta, d \bar{\zeta}]\left(\zeta_{3} d \zeta_{1} \wedge d \zeta_{2}+\zeta_{1} d \zeta_{2} \wedge d \zeta_{3}+\zeta_{2} d \zeta_{3} \wedge d \zeta_{1}\right)\right|_{\partial M_{\varrho}} \\
& \quad=\operatorname{Em}(\eta, \zeta) \sigma(d \zeta)
\end{aligned}
$$

Substituting (4.8), (4.9), (4.10) in (4.6) we obtain (2.2) together with the following formula for $\chi\left(k^{\prime}, \zeta\right)$ :

$$
\begin{aligned}
\chi\left(k^{\prime}, \zeta\right)= & 2 \pi i \int_{\eta \in \mathbb{R}^{3}} \theta\left(\gamma^{\prime} \eta\right) \frac{m(\eta, \zeta)}{((\bar{\zeta}-\zeta)(\zeta-\eta))^{2}} \delta\left(\eta^{2}-E\right) d \eta \\
& +\pi \int_{-\pi}^{\pi} \int_{0}^{\varrho} \frac{m(\eta(k(\tau), \varphi), \zeta)}{((\bar{\zeta}-\zeta)(\zeta-\eta(k(\tau), \varphi)))^{2}} d \tau d \varphi
\end{aligned}
$$

where $k^{\prime} \in \partial M_{\varrho}, \zeta \in \partial M_{r}, 0<\varrho \leq r ; \eta(k, \varphi), k(\tau), m(\eta, \zeta)$ are defined by (4.2c), (4.7), (4.11). $((\bar{\zeta}-\zeta)(\zeta-\eta))^{-2}$ is a continuously differentiable function on $\partial M_{r} \times M_{\varrho}$ for $\varrho<r$. It leads to the continuous differentiability of $\chi\left(k^{\prime}, \zeta\right)$ on $\partial M_{\varrho} \times \partial M_{r}$ for $\varrho<r$.

For the case $\varrho=r \quad \chi\left(k^{\prime}, \zeta\right)$ is a continuously differentiable function on $\left(\partial M_{\varrho} \times \partial M_{\varrho}\right) \backslash S_{\varrho}$, where $S_{\varrho}=\left\{\left(k^{\prime}, \zeta\right) \mid\left(k^{\prime}, \zeta\right) \in \partial M_{\varrho} \times \partial M_{\varrho}, \operatorname{Im} k^{\prime}=\operatorname{Im} \zeta\right\}$, and at fixed $k^{\prime} \chi\left(k^{\prime}, \zeta\right) \in L^{1}\left(\partial M_{\varrho}\right)$.

The result of Proposition 1B follows from (4.6), (4.8), (4.9). 


\section{The Proof of Proposition 2 and of Theorem 1}

Suppose that Proposition 2A is proved. Then the proof of Theorem 1A consists of the following. We multiply the right-hand side and the left-hand side of (2.3) by $(2 \pi)^{-3} e^{-i \ell x} v(x)$ and integrate it with respect to $x$. As a result we obtain (2.4).

The proof of Proposition 2A, using (2.2), is carried out according to the same scheme that the proof of (1.18), using (1.16), in [5, 6].

We substitute in (1.4) instead of $G$ its representation (2.2). Then (1.4) takes the form

$$
\begin{aligned}
\psi(x, k)= & e^{i k x}+\frac{1}{(2 \pi)^{3}} \int_{m \in \partial M_{r}} \int_{y \in \mathbb{R}^{3}} e^{i m(x-y)} \chi(k, m) \\
& \times v(y) \psi(y, k) d y \sigma(d m)+\int_{y \in \mathbb{R}^{3}} \frac{e^{i \sqrt{E}|x-y|}}{-4 \pi|x-y|} v(y) \psi(y, k) d y
\end{aligned}
$$

where $k^{2}=E, \sqrt{E}>0$. In addition, the two first terms in the right-hand side of (5.1) can be rewritten in the form

$$
\int_{m \in \partial M_{r}} Q^{+}(k, m) e^{i m x} \sigma(d m)
$$

where

$$
Q^{+}(k, m)=\delta(k-m)+\chi(k, m) h(k, m)
$$

Thus,

$$
\psi(x, k)=\int_{m \in \partial M_{r}} Q^{+}(k, m) e^{i m x} \sigma(d m)+\int_{y \in \mathbb{R}^{3}} \frac{e^{i \sqrt{E}|x-y|}}{-4 \pi|x-y|} v(y) \psi(y, k) d y .
$$

Comparing Eq. (5.2) for $\psi(x, k)$ and Eq. (1.15) for $\psi^{+}(x, k)$ we obtain

$$
\psi(x, k)=\int_{m \in \partial M_{r}} Q^{+}(k, m) \psi^{+}(x, m) \sigma(d m) .
$$

The proof of Proposition $2 \mathrm{~B}$ and of Theorem $1 \mathrm{~B}$ is carried out in a similar way. In proving Proposition $2 \mathrm{~B}$ we use (2.13) instead of (2.2).

\section{The Proof of Proposition 3.}

Proof of Lemma 3. Let $a$ be a fixed positive number such that $0<|\operatorname{Im} k|=\varrho<a$. Let $f(m, \ell)$ be a holomorphic function on $\bar{M}_{a} \times \bar{M}_{a}$. We shall prove that $\tilde{\Delta}(k)$ defined by (2.6) does not depend on $r$, where $\varrho<r \leq a$. We shall use the following equality

$$
\int_{|\operatorname{Im} m|=r_{1}} \chi(k, m) F(m) \sigma(d m)=\int_{|\operatorname{Im} m|=r_{2}} \chi(k, m) F(m) \sigma(d m)
$$

for any $F(m)$ from $\mathcal{O}\left(\bar{M}_{a}\right)$, where $\varrho<r_{1}, r_{2} \leq a$. 
If $F(m)=e^{\imath m x}$ then (6.1) is a consequence of (2.2). In the general case it is necessary to take into account the additional fact that any function from $\mathcal{O}\left(\bar{M}_{a}\right)$ admits an approximation by a linear combination of $e^{\imath m x}, x \in \mathbb{R}^{3}$.

Let $f(m, \ell)$ be a fixed holomorphic function on $\bar{M}_{a} \times \bar{M}_{a}$. Then $\tilde{\Delta}(k, \lambda)$ defined by (2.6) for $f_{\lambda}(m, \ell)=\lambda f(m, \ell)$ is a holomorphic function of $\lambda$.

Using this property of $\tilde{\Delta}$ (and standard arguments) it is sufficient to carry out the proof of Lemma 3 under the condition that $\|f\|_{C\left(\partial M_{a} \times \partial M_{a}\right)}$ is sufficiently small for the convergence of the series $-\sum_{j=1}^{\infty} \frac{P^{j}(k, r)}{j}$ to $\ln (I-P(k, r))$.

Now it is sufficient to prove that $\operatorname{tr} P^{j}(k, r)$ does not depend on $r$

$$
\begin{aligned}
\operatorname{tr} P^{j}(k, r)= & \int \ldots \int \chi\left(k, m_{j}\right) f\left(m_{j}, m_{j-1}\right) \ldots \\
& \times \ldots \chi\left(k, m_{2}\right) f\left(m_{2}, m_{1}\right) \chi\left(k, m_{1}\right) f\left(m_{1}, m_{j}\right) \\
& \times \sigma\left(d m_{1}\right) \ldots \sigma\left(d m_{j}\right) .
\end{aligned}
$$

Applying (6.1) to (6.2) with respect to each $m_{\imath}, i=1, \ldots, j$ we obtain that $\operatorname{tr} P^{j}(k, r)$ does not depend on $r$. Thus, Lemma 3 is proved.

Lemma 6. Let $f(m, \ell)$ be a holomorphic function on $\bar{M}_{r} \times \bar{M}_{r}$ and $\tilde{\Delta}(k)$ be defined by (2.6). Then

$$
\left.\bar{\partial}_{k} \tilde{\Delta}(k)\right|_{M_{r}}=\left.2 \pi \tilde{\Delta}(k) \int_{\xi \in \mathbb{R}^{3}}(\xi d \bar{k}) h(k+\xi, k+\xi) \delta\left(\xi^{2}+2 k \xi\right) d \xi\right|_{M_{r}}
$$

where $h(k, \ell)$ is determined by (2.4) (where $\ell \in M_{r}$ ).

It is sufficient to carry out the proof of Lemma 6 under the condition that $\tilde{\Delta}(k) \neq 0$. In this proof all $(0,1)$-forms are considered to be restricted to $M_{r}$.

We shall use that

$$
\bar{\partial}_{k} \int_{m \in \partial M_{r}} \chi(k, m) F(m) \sigma(d m)=-2 \pi \int_{\xi \in \mathbb{R}^{3}}(\xi d \bar{k}) \delta\left(\xi^{2}+2 k \xi\right) F(k+\xi) d \xi
$$

for any $F(m)$ from $\mathcal{O}\left(\bar{M}_{r}\right)$.

If $F(m)=e^{\imath m x}$ then (6.4) is a consequence of $\bar{\partial}$-equation for $G(x, k)$ and of representation of (2.2). It is necessary, in the general case, to take into account an additional fact that any function from $\mathcal{O}\left(\bar{M}_{r}\right)$ admits an approximation by a linear combination of $e^{i m x}, x \in \mathbb{R}^{3}$.

From (2.6) we obtain

$$
\bar{\partial}_{k} \ln \tilde{\Delta}(k)=\operatorname{Tr}\left((I-P(k, r))^{-1} \bar{\partial}_{k} P(k, r)\right), \quad|\operatorname{Im} k|<r .
$$

Denote by $\delta(\zeta-\eta)+R(\zeta, \eta ; k, r)$ the kernel of the integral operator $(I-P(k, r))^{-1}$. The function $f(k, \ell)$ is a holomorphic function on $\bar{M}_{r} \times \bar{M}_{r}$. As a consequence, $R(\ell, \eta ; k, r)$ and $h(k, \ell)$ are holomorphic functions on $\bar{M}_{r}$ with respect to $\ell$. So, in 
(6.6) we can use (6.4),

$$
\begin{aligned}
\operatorname{Tr} & \left((I-P(k, r))^{-1} \bar{\partial}_{k} P(k, r)\right) \\
= & \int_{m \in \partial M_{r}} \bar{\partial}_{k} \chi(k, m) f(m, m) \sigma(d m) \\
& +\int_{m \in \partial M_{r}} \int_{\eta \in \partial M_{r}} R(m, \eta ; k, r) \bar{\partial}_{k} \chi(k, m) f(m, \eta) \sigma(d \eta) \sigma(d m) \\
& \stackrel{(6.4)}{=}-2 \pi \int_{\xi \in \mathbb{R}^{3}}(\xi d \bar{k}) \delta\left(\xi^{2}+2 k \xi\right) f(k+\xi, k+\xi) d \xi \\
& +\int_{\xi \in \mathbb{R}^{3}} \int_{\eta \in \partial M_{r}}-2 \pi R(k+\xi, \eta ; k, r)(\xi d \bar{k}) \delta\left(\xi^{2}+2 k \xi\right) f(k+\xi, \eta) \sigma(d \eta) d \xi .
\end{aligned}
$$

Due to $(1.28)$

$$
R(\ell, \eta ; k+\xi, r)=R(\ell, \eta ; k, r), \quad \text { where } \xi \in \mathbb{R}^{3}, \xi^{2}+2 k \xi=0 .
$$

Due to (2.4) and (6.7),

$$
f(k+\xi, \ell)+\int_{\eta \in \partial M_{r}} R(\ell, \eta ; k, r) f(k+\xi, \eta) \sigma(d \eta)=h(k+\xi, \ell)
$$

where $\xi \in \mathbb{R}^{3}, \xi^{2}+2 k \xi=0$.

From (6.6) using (6.8) with $\ell=k+\xi$ we obtain

$$
\operatorname{Tr}\left((I-P(k, r))^{-1} \bar{\partial}_{k} P(k, r)\right)=-2 \pi \int_{\xi \in \mathbb{R}^{3}}(\xi d \bar{k}) h(k+\xi, k+\xi) \delta\left(\xi^{2}+2 k \xi\right) d \xi .
$$

From (6.5), (6.9) we obtain (6.3).

Proof of Proposition 3A. The Proof is based on the equalities (1.21), (1.27), Lemma 3 and Lemma 6 . Let $\alpha$ be a fixed positive number. Let the norm of the potential $v(x)$ be an infimum of $\beta$ such that (1.2) is held. Let $r$ be an arbitrary fixed positive number such that $r<\alpha / 2$. It is sufficient to prove the equality (2.7) for fixed $\alpha$ and $r$ and under the condition that the norm of the potential is sufficiently small in order that the following inequalities would be valid:

$$
|\Delta(k)-1|<1 / 2, \quad|\tilde{\Delta}(k)-1|<1 / 2 \text { on } M_{r} \backslash S^{2},
$$

where $S^{2}=\left\{k \mid k \in M_{r}, \operatorname{Im} k=0\right\}$.

In fact, suppose that for this case the equality (2.7) is proved. Consider an arbitrary real potential $v(x)$ with the property (1.2). Consider functions in the right-hand side and in the left-hand side of (2.7) for the family of the potentials $\lambda v(x)$, where $\lambda \in \mathbb{C}$. The functions $\Delta(k, \lambda), \Delta^{+}(k, \lambda), \exp \left[\lambda \hat{v}(0) \int_{m \in \partial M_{r}} \chi(k, m) \sigma(d m)\right]$ are entire functions of $\lambda$. The function $\tilde{\Delta}(k, \lambda)$ is an analytical function of $\lambda$ outside the set of zeros of $\Delta^{+}(k, \lambda)$. For $\lambda$ suffciently small in modulus the norm of $\lambda v(x)$ becomes sufficiently small in order that $(2.7)$ would be held by the assumption. It 
follows from analyticity with respect to $\lambda$ of the functions in the right-hand side and in the left-hand side of (2.7) that (2.7) is held for all $\lambda$ (where $\Delta^{+}(k, \lambda) \neq 0$ ) and, in particular, for the original potential $v(x)$.

Now it is sufficient to prove the equality (2.7) under condition $\left(^{*}\right)$. Due to this condition and due to Theorem 1A the function $h$ determined according to (1.5), (1.4) coincides with the function $h$ determined according to (2.4).

Denote by $\Delta^{\prime}(k)$ the right-hand side of (2.7). Using (1.27), (6.3), (6.4) (with $F(m) \equiv 1$ ) we obtain

$$
\left.\bar{\partial}_{k} \varphi(k)\right|_{M_{r}}=\left.\varphi A\right|_{M_{r}}, \quad \varphi=\Delta, \Delta^{\prime},
$$

where $\left.A\right|_{M_{r}}$ is the same $(0,1)$-form for $\Delta$ and $\Delta^{\prime}$.

Our following aim is to obtain (6.16). Let

$$
\zeta(\tau, k, \gamma)=\sqrt{1+\tau^{2} / E} k+i \tau \gamma,
$$

where $\tau \in \mathbb{R}, k$ and $\gamma$ are arbitrary fixed vectors such that

$$
k, \gamma \in \mathbb{R}^{3}, \quad k^{2}=E, \quad \gamma^{2}=1, \quad k \gamma=0 .
$$

(It follows from (6.11), (6.12) that $\zeta(\tau, k, \gamma) \in M_{r}$ for $\left.|\tau| \leq r\right)$. Due to (1.9) we have the equality

$$
\Delta(\zeta(+0, k, \gamma))=\Delta_{\gamma}(k)
$$

In addition (see Lemma 3 and its proof) the following equality is valid:

$$
\tilde{\Delta}_{\gamma}(k)=\tilde{\Delta}(\zeta(+0, k, \gamma))
$$

where $\tilde{\Delta}_{\gamma}$ and $\tilde{\Delta}$ are defined by (1.20), (2.6) respectively;

$$
2 \pi i \int_{m \in \mathbb{R}^{3}} \theta((m-k) \gamma) \delta\left(k^{2}-m^{2}\right) d m=\int_{m \in \partial M_{r}} \chi(\zeta(+0, k, \gamma), m) \sigma(d m) .
$$

From (1.21), (6.13), (6.14), (6.15) we obtain

$$
\Delta(\zeta(+0, k, \gamma))=\Delta^{\prime}(\zeta(+0, k, \gamma)) .
$$

The final part of the proof of Proposition 3A consists of the following: Let $k^{\prime}$ be a fixed point in $M_{r}, \operatorname{Im} k^{\prime} \neq 0$. Let be $n=\operatorname{Re} k^{\prime} \times \operatorname{Im} k^{\prime}$. We consider one-dimensional complex manifold

$$
V=\left\{k \mid k \in M_{r}, k n=0, \operatorname{det}[\operatorname{Re} k, \operatorname{Im} k, n] \geq 0\right\} .
$$

(It is obvious that $k^{\prime} \in V$.) $V$ is isomorphic to the ring in the complex plane $\partial V=S_{1} \cup S_{2}$, where $S_{1} \in S^{2}=\left\{k \mid k \in M_{r}, \operatorname{Im} k=0\right\}$.

In view of (6.16) we have

$$
\left.\left(\left.\Delta(k)\right|_{V}\right)\right|_{S_{1}}=\left.\left(\left.\Delta^{\prime}(k)\right|_{V}\right)\right|_{S_{1}} .
$$

Under restriction to $V, A$ is $(0,1)$-form with the continuous coefficient.

Due to (6.17) and in view of Eq. (6.10) restricted, in addition, to $V$, we obtain $\left.\Delta(k)\right|_{V}=\left.\Delta^{\prime}(k)\right|_{V}$ and, in particular, $\Delta\left(k^{\prime}\right)=\Delta^{\prime}\left(k^{\prime}\right)$.

The proof of Proposition $3 \mathrm{~A}$ is fulfilled. 
The proof of Lemma 5 is similar to the proof of Lemma 3. In this proof, instead of (6.1) we use that

$$
\int_{m \in R(k)} s(k, m)(|m|)^{-1} F(m) \sigma(d m)=\int_{m \in \partial M_{r}} \chi(k, m) F(m) \sigma(d m)
$$

for any $F(m)$ from $\mathcal{O}\left(\bar{M}_{a}\right)$, where $0<|\operatorname{Im} k| \leq r \leq a$. (In the left-hand side of (6.18) $\sigma(d m)$ is a surface element, in the right-hand side of (6.18) $\sigma(d m)$ is an element of the volume).

Proposition $3 \mathrm{~B}$ is a consequence of Proposition 3A, Lemma 5 and the equality (6.18) (for $F(m)=1$ ).

Propositions $4 \mathrm{~A}$ and $4 \mathrm{~B}$ are consequences of Propositions $3 \mathrm{~A}$ and $3 \mathrm{~B}$ [and of elementary properties of Eqs. (2.4), (2.15)].

\section{Appendix to the Proof of Theorem 2}

The aim of this appendix is to prove the estimate (3.3).

Let $k(\lambda)$ be defined by (3.1), then the formulas (7.1)-(7.4) are valid:

$$
\begin{aligned}
& d \bar{k}_{1}=\frac{\sqrt{E_{\tau}}\left(\bar{\lambda}^{2}-1\right)}{2 \bar{\lambda}^{2}} d \bar{\lambda}, \quad d \bar{k}_{2}=\frac{i \sqrt{E_{\tau}}\left(\bar{\lambda}^{2}+1\right)}{2 \bar{\lambda}^{2}} d \bar{\lambda}, \quad d \bar{k}_{3}=0 ; \\
& \operatorname{Re} k_{1}=\frac{\sqrt{E_{\tau}}(\lambda+\bar{\lambda})(\lambda \bar{\lambda}+1)}{4 \lambda \bar{\lambda}}, \quad \operatorname{Re} k_{2}=\frac{\sqrt{E_{\tau}}(\lambda-\bar{\lambda})(\lambda \bar{\lambda}+1)}{4 i \lambda \bar{\lambda}} \text {, } \\
& \operatorname{Re} k_{3}=\frac{\tau}{2} \\
& \operatorname{Im} k_{1}=\frac{\sqrt{E_{\tau}}(\lambda-\bar{\lambda})(\lambda \bar{\lambda}-1)}{4 i \lambda \bar{\lambda}}, \quad \operatorname{Im} k_{2}=\frac{\sqrt{E_{\tau}}(\lambda+\bar{\lambda})(\lambda \bar{\lambda}-1)}{-4 \lambda \bar{\lambda}} \text {, } \\
& \operatorname{Im} k_{3}=0 ; \quad|\operatorname{Im} k|=\frac{\sqrt{E_{\tau}}(\lambda \bar{\lambda}-1)}{2 \sqrt{\lambda \bar{\lambda}}}, \quad \text { for } \quad|\lambda| \geq 1 \text {. } \\
& \frac{(\operatorname{Re} k \times \operatorname{Im} k)_{1}}{|\operatorname{Im} k|}=\frac{\tau(\lambda+\bar{\lambda})}{4 \sqrt{\lambda \bar{\lambda}}}, \quad \frac{(\operatorname{Re} k \times \operatorname{Im} k)_{2}}{|\operatorname{Im} k|}=\frac{\tau(\lambda-\bar{\lambda})}{4 i \sqrt{\lambda \bar{\lambda}}}, \\
& \frac{(\operatorname{Re} k \times \operatorname{Im} k)_{3}}{|\operatorname{Im} k|}=\frac{\sqrt{E_{\tau}}(1+\lambda \bar{\lambda})}{-2 \sqrt{\lambda \bar{\lambda}}}, \text { for }|\lambda| \geq 1 \text {. }
\end{aligned}
$$

Using the formula

$$
\left.\bar{\partial}_{k} g\right|_{k^{2}=E}=\left.e^{-i k x} \bar{\partial}_{k} G\right|_{k^{2}=E}
$$

and formulas (4.2), (7.1)-(7.4) we obtain

$$
\bar{\partial}_{\lambda} g(k, \lambda, \bar{\lambda})=\frac{e^{-i \operatorname{Re} k x}}{-32 \pi^{2}}\left(\tilde{I}_{1} \frac{\sqrt{E_{\tau}}(\lambda \bar{\lambda}+1)}{\bar{\lambda} \sqrt{\lambda \bar{\lambda}}}+\tilde{I}_{2} \frac{\tau}{\bar{\lambda}}\right) d \bar{\lambda}
$$


where $k(\lambda)$ is defined by (3.1), $|\lambda| \geq 1$,

$$
\begin{aligned}
& \tilde{I}_{1}=\int_{-\pi}^{\pi}(\cos \varphi-1) \exp \left(\cos \varphi \operatorname{Re} k x+\sin \varphi \frac{\operatorname{Re} k \times \operatorname{Im} k}{|\operatorname{Im} k|} x\right) d \varphi, \\
& \tilde{I}_{2}=\int_{-\pi}^{\pi}(\sin \varphi) \exp \left(\cos \varphi \operatorname{Re} k x+\sin \varphi \frac{\operatorname{Re} k \times \operatorname{Im} k}{|\operatorname{Im} k|} x\right) d \varphi .
\end{aligned}
$$

Replace $\bar{\lambda}$ in (7.2), (7.4) by an independent complex variable $\zeta$. Then $\left(\operatorname{Re} k_{j}\right)(\lambda, \zeta)$, $\left(\frac{(\operatorname{Re} k \times \operatorname{Im} k)_{j}}{|\operatorname{Im} k|}\right)(\lambda, \zeta)$ are holomorphic functions in the domain (3.2). In this domain the imaginary part of each of these functions admits the estimate

$$
|\operatorname{Im} F(\lambda, \zeta)|<\text { const } \cdot|\zeta-\bar{\lambda}| \text {. }
$$

First of all, we shall prove (7.7) for $F=\left(|\operatorname{Im} k|^{-1}(\operatorname{Re} k \times \operatorname{Im} k)_{3}\right)$. In this case it is sufficient to prove (7.7) for $F(\lambda, \zeta)=(\lambda \zeta)^{ \pm 1 / 2}$. Let $\lambda=r e^{i \alpha}, \zeta=R e^{i \beta}$, $\psi=\alpha+\beta, A=r R$, where in view of (3.2) $r \geq 1, R \geq 1,-\pi / 2 \leq \alpha+\beta \leq \pi / 2$. The needed estimate is a consequence of the following sequence of equalities and of inequalities:

$$
\begin{aligned}
\left|\operatorname{Im}(\lambda \zeta)^{-1 / 2}\right| & =\left|A^{-1 / 2} \sin (\psi / 2)\right| \leq\left|\operatorname{Im}(\lambda \zeta)^{1 / 2}\right|=\left|A^{1 / 2} \sin (\psi / 2)\right| \\
& \leq \sqrt{2 A-2 A \cos \psi} \leq \sqrt{R^{2}+r^{2}-2 R r \cos \psi}=|\zeta-\bar{\lambda}|
\end{aligned}
$$

For $F=\left(|\operatorname{Im} k|^{-1}(\operatorname{Re} k \times \operatorname{Im} k)_{1}\right)(7.7)$ is a consequence of the formula

$$
\begin{aligned}
\left|\operatorname{Im} \frac{(\lambda+\zeta)}{\sqrt{\lambda \zeta}}\right| & =\left|\operatorname{Im} \frac{(\lambda+\bar{\lambda})+(\zeta-\bar{\lambda})}{\sqrt{\lambda \zeta}}\right| \\
& \leq 2\left(\frac{r}{R}\right)^{1 / 2}|\sin (\psi / 2)|+\frac{|\zeta-\bar{\lambda}|}{\sqrt{R r}} \leq 3|\zeta-\bar{\lambda}| .
\end{aligned}
$$

The case of $F=\left(|\operatorname{Im} k|^{-1}(\operatorname{Re} k \times \operatorname{Im} k)_{2}\right)$ is similar to the previous case. For $F=\left(\operatorname{Re} k_{1}\right)(7.7)$ is a consequence of the formula

$$
\begin{aligned}
\left|\operatorname{Im} \frac{(\lambda+\zeta)(\lambda \zeta+1)}{\lambda \zeta}\right| & =\left|\operatorname{Im}\left(\lambda+\bar{\lambda}+(\zeta-\bar{\lambda})+\frac{\lambda+\bar{\lambda}+(\zeta-\bar{\lambda})}{\lambda \zeta}\right)\right| \\
& \leq|\zeta-\bar{\lambda}|+\frac{2}{R}|\sin \psi|+\frac{|\zeta-\bar{\lambda}|}{R r} \leq 6|\zeta-\bar{\lambda}| .
\end{aligned}
$$

The case $F=\left(\operatorname{Re} k_{2}\right)$ is similar to the previous case.

For the case $F=\left(\operatorname{Re} k_{3}\right)$ the estimate (7.7) is obvious.

In the domain (3.2) the function $g(x, \lambda, \zeta)$ is defined by the formula

$$
g(x, \lambda, \zeta)=g(x, \lambda, \bar{\lambda})+\int_{\bar{\lambda}}^{\zeta} \partial_{\bar{\eta}} g(x, \lambda, \bar{\eta})
$$

Using (7.8), (7.6) and the estimates given above we obtain (3.3). 


\section{The Uniform Estimate of the Green Function $g(x, k)$ at Fixed $k^{2}=E$ for $|k| \rightarrow \infty$}

Let $E \in \mathbb{R}$ be fixed. Let $\eta$ and $\gamma$ be fixed such that $\gamma, \eta \in \mathbb{R}^{3}, \gamma^{2}=\eta^{2}=1, \gamma \eta=0$. Let

$$
D(\gamma, \varepsilon)=\left\{x\left|x \in \mathbb{R}^{3},\right| x \gamma|\geq \varepsilon,| x-\gamma(x \gamma) \mid \geq \varepsilon\right\} \quad \text { for } \varepsilon>0 .
$$

Consider a real curve

$$
k(\tau)=\eta \sqrt{E+\tau^{2}}+i \gamma \tau, \quad \begin{cases}\tau>0 & \text { for } E \geq 0 \\ \tau>\sqrt{-E} & \text { for } E<0\end{cases}
$$

We have $k(\tau) \subset M=\left\{k \mid k \in \mathbb{C}^{3}, k^{2}=E\right\}$.

Lemma 7. For any $\varepsilon>0$,

$$
\max _{x \in D(\gamma, \varepsilon)} g(x, k(\tau)) \rightarrow 0 \text { for } \tau \rightarrow \infty
$$

Let be $v(x)(1+|x|)^{3+\varepsilon} \in L^{\infty}\left(\mathbb{R}^{3}\right)$, then from Lemma 1 and Lemma 7 it follows that

$$
\begin{aligned}
\max _{x \in \mathbb{R}^{3}} \int_{y \in \mathbb{R}^{3}}|g(x-y, k) v(y)| d y \rightarrow 0 & \text { for } \quad k^{2}=E,|k| \rightarrow \infty, \\
\|B(x, y ; k)\|_{L^{2}\left(\mathbb{R}^{3} \times \mathbb{R}^{3}\right)} \rightarrow 0 & \text { for } \quad k^{2}=E,|k| \rightarrow \infty,
\end{aligned}
$$

where $B(x, y ; k)$ is a kernel of the integral operator $B(k)$ from (1.7). The formulas (1.29), (1.30) follow from $(*)$. The formula (1.31) follows from $(* *)$.

We shall give a proof of Lemma 7 at $E \geq 0$. It is sufficient to carry out the proof of Lemma 7 for

$$
\gamma=(0,1,0)
$$

We shall carry out some calculations:

$$
\begin{aligned}
-(2 \pi)^{3} g(x, k) & =\int_{\xi \in \mathbb{R}^{3}} \frac{e^{i \xi x} d \xi}{\xi^{2}+2 k \xi}=\int_{\xi \in \mathbb{R}^{3}} \frac{e^{i \xi x} d \xi}{(\xi+\operatorname{Re} k)^{2}-(\operatorname{Re} k)^{2}+2 i \operatorname{Im} k \xi} \\
& =\int_{\xi^{\prime} \in \mathbb{R}^{3}} \frac{e^{i\left(\xi^{\prime}-\operatorname{Re} k\right) x} d \xi^{\prime}}{\left(\xi^{\prime}\right)^{2}-(\operatorname{Re} k)^{2}+2 i \operatorname{Im} k\left(\xi^{\prime}-\operatorname{Re} k\right)} \\
& =e^{-i \operatorname{Re} k x} \int_{\xi \in \mathbb{R}^{3}} \frac{e^{\imath \xi x} d \xi}{\xi^{2}-(\operatorname{Re} k)^{2}-2 i \operatorname{Im} k \operatorname{Re} k+2 i \operatorname{Im} k \xi} \\
& =e^{-\imath \operatorname{Re} k x} \int_{\xi \in \mathbb{R}^{3}} \frac{e^{i \xi x} d \xi}{\xi^{2}+2 i \operatorname{Im} k \xi-k^{2}-(\operatorname{Im} k)^{2}} \\
& =e^{-i \operatorname{Re} k x} J(x, k) .
\end{aligned}
$$

Due to (8.2), (8.3) we have further

$$
J(x, k)=\iint e^{i\left(\xi_{1} x_{1}+\xi_{3} x_{3}\right)} I\left(\xi_{1}, \xi_{3}, x_{2}, E, \tau\right) d \xi_{1} d \xi_{3},
$$


where

$$
\begin{aligned}
I\left(\xi_{1}, \xi_{3}, x_{2}, E, \tau\right) & =\int \frac{e^{i \xi_{2} x_{2}} d \xi_{2}}{\xi_{2}^{2}+2 i \xi_{2} \tau+\xi_{1}^{2}+\xi_{3}^{2}-\left(\tau^{2}+E\right)}, \\
\xi & =\left(\xi_{1}, \xi_{2}, \xi_{3}\right) \in \mathbb{R}^{3} .
\end{aligned}
$$

The equation

$$
\xi_{2}^{2}+2 i \xi_{2} \tau+\xi_{1}^{2}+\xi_{3}^{2}-\left(\tau^{2}+E\right)=0
$$

has the following roots with respect to $\xi_{2} \in \mathbb{C}$ :

$$
\xi_{2}=-i \tau \pm \sqrt{E-\xi_{1}^{2}-\xi_{3}^{2}} .
$$

The following proof of Lemma 7 [under the condition (8.3)] splits into two cases: $x_{2}>0$ and $x_{2}<0$.

A) Let $x_{2}>0$, then

$$
\begin{aligned}
I\left(\xi_{1}, \xi_{3}, x_{2}, E, \tau\right)= & 0 \\
& \text { for } \xi_{1}^{2}+\xi_{3}^{2}<E+\tau^{2}, \\
I\left(\xi_{1}, \xi_{3}, x_{2}, E, \tau\right)= & 2 \pi i \frac{\exp \left[i\left(-i \tau+i \sqrt{\xi_{1}^{2}+\xi_{3}^{2}-E}\right) x_{2}\right]}{2 i \sqrt{\xi_{1}^{2}+\xi_{3}^{2}-E}} \\
& \text { for } E+\tau^{2}<\xi_{1}^{2}+\xi_{3}^{2} .
\end{aligned}
$$

Thus

$$
\begin{aligned}
J & =\pi e^{\tau x_{2}} \iint_{E+\tau^{2}<\xi_{1}^{2}+\xi_{3}^{2}} e^{i\left(\xi_{1} x_{1}+\xi_{3} x_{3}\right)} \frac{\exp \left[-\sqrt{\xi_{1}^{2}+\xi_{3}^{2}-E} x_{2}\right]}{\sqrt{\xi_{1}^{2}+\xi_{3}^{2}-E}} d \xi_{1} d \xi_{3} \\
& =\pi e^{\tau x_{2}} \int_{-\pi}^{\pi} e^{i r \varrho \cos \varphi} \int_{\sqrt{E+\tau^{2}}}^{+\infty} \frac{\exp \left[-\sqrt{r^{2}-E} x_{2}\right]}{\sqrt{r^{2}-E}} r d r d \varphi \\
& =\pi e^{\tau x_{2}} \int_{-\pi}^{\pi} e^{i r \varrho \cos \varphi} \int_{\sqrt{E+\tau^{2}}}^{+\infty} \exp \left[-\sqrt{r^{2}-E} x_{2}\right] d \sqrt{r^{2}-E} d \varphi \\
& =\pi e^{\tau x_{2}} \int_{-\pi}^{\pi} \exp \left[i \sqrt{s^{2}+E} \varrho \cos \varphi\right] \int_{\tau}^{+\infty} e^{-s x_{2}} d s d \varphi \\
& =\pi e^{\tau x_{2}} \int_{\tau}^{+\infty} e^{-s x_{2}} \int_{-\pi}^{\pi} \exp \left[i \sqrt{s^{2}+E} \varrho \cos \varphi\right] d \varphi d s .
\end{aligned}
$$

From here we obtain

$$
|J| \leq e^{\tau x_{2}} \int_{\tau}^{+\infty} e^{-s x_{2}} \frac{\text { const }}{\sqrt{1+\varrho \sqrt{s^{2}+E}}} d s \leq \frac{\text { const }}{x_{2} \sqrt{1+\varrho \sqrt{\tau^{2}+E}}},
$$

where $\varrho=\sqrt{x_{1}^{2}+x_{3}^{2}}$. 
B) Let be $x_{2}<0$, then

$$
\begin{aligned}
& I\left(\xi_{1}, \xi_{3}, x_{2}, E, \tau\right)=-2 \pi i\left[\frac{\exp \left[-\tau\left|x_{2}\right|-i \sqrt{E-\xi_{1}^{2}-\xi_{3}^{2}}\left|x_{2}\right|\right]}{2 \sqrt{E-\xi_{1}^{2}-\xi_{3}^{2}}}\right. \\
& \left.-\frac{\exp \left[-\tau\left|x_{2}\right|+i \sqrt{E-\xi_{1}^{2}-\xi_{3}^{2}}\left|x_{2}\right|\right]}{2 \sqrt{E-\xi_{1}^{2}-\xi_{3}^{2}}}\right] \\
& =-2 \pi e^{-\tau\left|x_{2}\right|} \frac{\sin \left(\sqrt{E-\xi_{1}^{2}-\xi_{3}^{2}}\left|x_{2}\right|\right)}{\sqrt{E-\xi_{1}^{2}-\xi_{3}^{2}}} \\
& \text { for } \xi_{1}^{2}+\xi_{3}^{2}<E \text {, } \\
& I\left(\xi_{1}, \xi_{3}, x_{2}, E, \tau\right)=-2 \pi i\left[\frac{\exp \left[-\tau\left|x_{2}\right|+\sqrt{\xi_{1}^{2}+\xi_{3}^{2}-E}\left|x_{2}\right|\right]}{2 i \sqrt{\xi_{1}^{2}+\xi_{3}^{2}-E}}\right. \\
& \left.-\frac{\exp \left[-\tau\left|x_{2}\right|-\sqrt{\xi_{1}^{2}+\xi_{3}^{2}-E}\left|x_{2}\right|\right]}{2 i \sqrt{\xi_{1}^{2}+\xi_{3}^{2}-E}}\right] \\
& \text { for } E<\xi_{1}^{2}+\xi_{3}^{2}<E+\tau^{2} \text {, } \\
& I\left(\xi_{1}, \xi_{3}, x_{2}, E, \tau\right)=\pi \frac{\exp \left[-\tau\left|x_{2}\right|-\sqrt{\xi_{1}^{2}+\xi_{3}^{2}-E}\left|x_{2}\right|\right]}{\sqrt{\xi_{1}^{2}+\xi_{3}^{2}-E}} \\
& \text { for } E+\tau^{2}<\xi_{1}^{2}+\xi_{3}^{2} \text {; }
\end{aligned}
$$

thus

$$
J=J_{1}+J_{2}+J_{3},
$$

where

$$
\begin{aligned}
J_{1} & =-2 \pi e^{-\tau\left|x_{2}\right|} \iint_{\xi_{1}^{2}+\xi_{3}^{2}<E} e^{i\left(\xi_{1} x_{1}+\xi_{3} x_{3}\right)} \frac{\sin \left(\sqrt{E-\xi_{1}^{2}-\xi_{3}^{2}}\left|x_{2}\right|\right)}{\sqrt{E-\xi_{1}^{2}-\xi_{3}^{2}}} d \xi_{1} d \xi_{3}, \\
\left|J_{1}\right| & \leq \text { const } e^{-\tau\left|x_{2}\right|} \int_{0}^{\sqrt{E}} \frac{r d r}{\sqrt{E-r^{2}}}=\operatorname{const} \sqrt{E} e^{-\tau\left|x_{2}\right|} ; \\
J_{2} & =-\pi e^{-\tau\left|x_{2}\right|} \int_{E<\xi_{1}^{2}+\xi_{3}^{2}<E+\tau^{2}} e^{i\left(\xi_{1} x_{1}+\xi_{3} x_{3}\right)} \frac{\exp \left[\sqrt{\xi_{1}^{2}+\xi_{3}^{2}-E}\left|x_{2}\right|\right]}{\sqrt{\xi_{1}^{2}+\xi_{3}^{2}-E}} d \xi_{1} d \xi_{3} \\
& =-\pi e^{-\tau\left|x_{2}\right|} \int_{-\pi}^{\pi} e^{i r \varrho \cos \varphi} \int_{-\pi}^{\sqrt{E+\tau^{2}}} \frac{\exp \left[\sqrt{r^{2}-E}\left|x_{2}\right|\right]}{\sqrt{r^{2}-E}} r d r d \varphi \\
& =-\pi e^{-\tau\left|x_{2}\right|} \int_{-\pi}^{\pi} \exp \left[i \sqrt{s^{2}+E} \varrho \cos \varphi\right] \int_{0}^{s\left|x_{2}\right|} d s d \varphi \\
& =-\pi e^{-\tau\left|x_{2}\right|} \int_{0}^{\tau} e^{s\left|x_{2}\right|} \int_{-\pi}^{\pi} \exp \left[i \sqrt{s^{2}+E} \varrho \cos \varphi\right] d \varphi d s
\end{aligned}
$$




$$
\begin{aligned}
\left|J_{2}\right| & \leq e^{-\tau\left|x_{2}\right|} \int_{0}^{\tau} e^{s\left|x_{2}\right|} \frac{\text { const }}{\sqrt{1+\varrho \sqrt{s^{2}+E}}} d s \\
& \triangleq e^{-\tau\left|x_{2}\right|}\left(\int_{0}^{\sqrt{\tau}} e^{s\left|x_{2}\right|} \frac{\text { const }}{\sqrt{1+\varrho \sqrt{E}}} d s+\int_{\sqrt{\tau}}^{\tau} e^{s\left|x_{2}\right|} \frac{\text { const }}{\sqrt{1+\varrho \sqrt{\tau+E}}} d s\right) \\
& \leq \operatorname{const} \frac{e^{-\tau\left|x_{2}\right|}}{\left|x_{2}\right|}\left(e^{\sqrt{\tau}\left|x_{2}\right|}+\frac{e^{\tau\left|x_{2}\right|}}{\sqrt{1+\varrho \sqrt{\tau+E}}}\right) \\
& =\operatorname{const} \frac{1}{\left|x_{2}\right|}\left(\exp \left[(\sqrt{\tau}-\tau)\left|x_{2}\right|\right]+\frac{1}{\sqrt{1+\varrho \sqrt{\tau+E}}}\right)
\end{aligned}
$$

where $\varrho=\sqrt{x_{1}^{2}+x_{3}^{2}}, \tau>1$;

$$
\begin{aligned}
J_{3} & =\pi e^{-\tau\left|x_{2}\right|} \iint_{E<\xi_{1}^{2}+\xi_{3}^{2}} e^{\imath\left(\xi_{1} x_{1}+\xi_{3} x_{3}\right)} \frac{\exp \left[-\sqrt{\xi_{1}^{2}+\xi_{3}^{2}-E}\left|x_{2}\right|\right]}{\sqrt{\xi_{1}^{2}+\xi_{3}^{2}-E}} d \xi_{1} d \xi_{3} \\
& =\pi e^{-\tau\left|x_{2}\right|} \int_{-\pi}^{\pi} e^{\imath r \varrho \cos \varphi} \int_{\sqrt{E}}^{+\infty} \frac{\exp \left[\sqrt{r^{2}-E}\left|x_{2}\right|\right]}{\sqrt{r^{2}-E}} r d r d \varphi \\
& =\pi e^{-\tau\left|x_{2}\right|} \int_{-\pi}^{\pi} e^{i \sqrt{s^{2}+E} \varrho \cos \varphi} \int_{0}^{+\infty} e^{-s\left|x_{2}\right|} d s \\
\left|J_{3}\right| & \leq \text { const } \frac{e^{-\tau\left|x_{2}\right|}}{\left|x_{2}\right|} .
\end{aligned}
$$

Thus, under our assumption (8.3) the result of Lemma 7 follows from (8.4), (8.7) for $x_{2}>0$ and from (8.4), (8.8), (8.9), (8.10), (8.11) for $x_{2}<0$.

\section{References}

1. Beals, R., Coifman, R.R.: Multidimensional inverse scattering and nonlinear partial differential equations. Proc. Symp. Pure Math. 43, 45-70 (1985)

2. Chadan, K., Sabatier, P.C.: Inverse problem in quantum scattering theory. $2^{\text {nd }}$ ed. Berlin, Heidelberg, New York: Springer 1989

3. Ehrenpreis, L.: Fourier analysis in several complex variables. New York: Wiley 1970

4. Faddeev, L.D.: Growing solutions of the Schrödinger equation. Dokl. Akad. Nauk SSSR 165, 514-517 (1965), translation in Sov. Phys. Dokl. 10, 1033-1035 (1966)

5. Faddeev, L.D.: Factorization of the $S$ matrix for the multidimensional Schrödinger operator. Dokl. Akad. Nauk SSSR 167, 69-72 (1966), translation in Sov. Phys. Dokl. 11, 209-211 (1966)

6. Faddeev, L.D.: Inverse problem of quantum scattering theory. II. Itogi Nauki i Tekhniki, Sov. Prob. Mat. 3, 93-180 (1974), translation in J. Sov. Math. 5, 334-396 (1976)

7. Gel'fand, I.M.: Some problems of functional analysis and algebra. In: Proceedings of the International Congress of Mathematicians, 1954, held at Amsterdam

8. Gohberg, I.C., Krein, M.G.: Introduction to the theory of linear nonselfadjoint operators. Moscow: Nauka 1965 
9. Henkin, G.M.: Continuation of bounded holomorphic functions from submanifolds in general position to strictly pseudoconvex domains. Izvestija Akad. Nauk SSSR. Ser. Mat. 36, 540-567 (1972)

10. Nachman, A.I., Sylvester, J., Uhlmann, G.: An n-dimensional Borg-Levinson theorem. Commun. Math. Phys. 115, 595-605 (1988)

11. Nachman, A.I., Ablowitz, M.J.: A multidimensional inverse scattering method. Studies in Appl. Math. 71, 243-250 (1984)

12. Nachman, A.I.: Reconstructions from boundary measurements. Ann. Math. 128, 531-576 (1988)

13. Newton, R.G.: Scattering theory of waves and particles. $2^{\text {nd }}$ ed. Berlin, Heidelberg, New York: Springer 1982

14. Novikov, R.G.: Reconstruction of a two-dimensional Schrödinger operator from the scattering amplitude at fixed energy. Funkt. Anal. i Pril. 20 (3), 90-91 (1986), translation in Funct. Anal. and Appl. 20, 246-248 (1986)

15. Novikov, R.G., Henkin, G.M.: The $\bar{\partial}$-equation in the multidimensional inverse scattering problem. a) Preprint 27M, Institute of Physics, Krasnoyarsk (1986) (in russian); b) Uspekhi Mat. Nauk 42 (3), 93-152 (1987), translation in Russ. Math. Surv. 42 (4), 109-180 (1987)

16. Novikov, R.G.: Multidimensional inverse spectral problem for the equation $-\Delta \psi+(v(x)-$ $E u(x)) \psi=0$. Funkt. Anal. i Pril. 22 (4), 11-22 (1988), translation in Funct. Anal. and Appl. 22 (4), 263-272 (1988)

17. Novikov, R.G.: The inverse scattering problem on a fixed energy level for the two-dimensional Schrödinger operator. J. Funct. Anal. 103, 409-463 (1992)

18. Novikov, R.G.: Reconstruction of an exponentially decreasing potential for the three-dimensional Schrödinger equation through the scattering amplitude at fixed energy. C.R. Acad. Sci. Paris 316, Sér. I, 657-662 (1993)

19. Stout, E.L.: An integral formula for holomorphic functions on strictly pseudoconvex hypersurfaces. Duke Math. J. 42, 347-356 (1975)

20. Sylvester, J., Uhlmann, G.: A global uniqueness theorem. Ann. Math. 125, 153-169 (1987)

21. Sylvester, J., Uhlmann, G.: The Dirichlet to Neumann map and applications in Inverse Problems in PDE. Arcata, July-August 1989, edited by D. Colton, R. Ewing and W. Rundell, SIAM Proceeding, 1990 pp. 101-139

22. Kohn, R., Vogelius, M.: Determining conductivity by boundary measurements. II. Interior results. Comm. Pure Appl. Math. 38, 644-667 (1985)

Communicated by A. Jaffe 
